\title{
General second-rank correlation tensors for homogeneous magnetohydrodynamic turbulence
}

\author{
S. Oughton \\ Department of Mathematics, University College London, London WC1E 6BT, United Kingdom \\ K.-H. Rädler \\ Astrophysikalisches Institut Potsdam, An der Sternwarte 16, D-14482 Potsdam, Germany
}

W. H. Matthaeus

Bartol Research Institute, University of Delaware, Newark, Delaware 19716

(Received 16 January 1997; revised manuscript received 29 April 1997)

\begin{abstract}
The properties and structure of second-order (Cartesian) correlation tensors are derived for the general case of two solenoidal random vector fields. The theory is intended to describe homogeneous magnetohydrodynamic turbulence, with no assumed rotational or reflectional symmetries. Each correlation tensor can be written in terms of four scalar generating functions and the relationship of these functions to the potentials that generate the poloidal and toroidal components of the underlying vector fields is derived. The physical nature of the scalar functions is investigated and their true or pseudoscalar character is ascertained. In our general discussion we clarify several misleading statements dating back to Robertson's original paper in the field [Proc. Camb. Philos. Soc. 36, 209 (1940)]. It is also shown that using the one-dimensional correlation function, it is possible to obtain spectral information on the induced electric field in directions perpendicular to the measurement direction. [S1063-651X(97)09208-8]
\end{abstract}

PACS number(s): 47.27.Gs, 52.65.Kj, 47.65.+a

\section{INTRODUCTION}

Turbulence theory makes extensive use of a general technique, attributed to Robertson [1], for a complete and compact representation of correlation functions associated with a random vector field, such as the velocity field fluctuation in homogeneous turbulence [2]. This theory of invariant tensor structure usually has been applied to highly symmetric situations, most often isotropic turbulence [1-3], and for some years has remained a key element in the exposition of basic turbulence theory $[4,5]$. The present paper is motivated by the need for a clear exposition of the general form of Cartesian correlation functions involving two distinct solenoidal vector fields, say a magnetic field and an incompressible velocity field, with no additional assumptions other than spatial homogeneity of the second-order correlations.

While isotropy has been the most frequent assumption, there have been extensive discussions of the structure of correlation tensors for axisymmetric turbulence, both for the mirror-symmetric [6,7] and non-mirror-symmetric cases [8]. To our knowledge, correlation structure for the full set of second-order correlations, involving both velocity and magnetic fields, has been given previously only for the isotropic case $[9,10]$, although various special cases enter into meanfield dynamo theory (see, e.g., [11]).

Non-mirror-symmetric turbulent fields are essential elements in discussions of the role of magnetic helicity in dynamo theory $[11,12]$ and in the theory of magnetohydrodynamic (MHD) cascades [13] and relaxation [14]. However, most treatments of helicity have again focused on isotropic fluctuations, in this case allowing for index-antisymmetric contributions to the correlation tensors. The general result for the structure of the antisymmetric autocorrelation tensor, for arbitrary rotational symmetry, was presented in the context of solar wind observations [15]

For hydrodynamics, isotropy and mirror symmetry remain reasonable approximations in many situations; however, anisotropy is expected to become significant in a variety of circumstances. Preferred directions, such as might be associated with rotation, or a large-scale gradient can have impact on locally homogeneous turbulence, and representation of the correlation tensors must allow for this possibility $[7,16]$. Homogeneous turbulence can also depend on higher-order tensor quantities, such as the gradient tensor of a nonuniform mean flow, (see, e.g., [17]), although we do not consider such extensions here. In many cases symmetries with respect to tensor quantities and preferred directions have direct impact on the structure of the correlation tensors. This is especially true for MHD turbulence for which, in many physical applications, there may be an influential local mean magnetic field direction that can induce spectral and spatial correlation anisotropy. (For a review of the extensively studied example of anisotropic turbulence in solar wind fluctuations, see [18].) While axisymmetric representations may be adequate in some cases, there are clear motivations to go a step further and investigate the most general two-point, two-field correlations for incompressible homogeneous turbulence. For example, it is not uncommon to be presented simultaneously with two preferred directions, such as in the solar wind, with a mean magnetic field direction and a direction (heliocentric radial) associated with mean large-scale gradients. Just as important, it turns out that the most general case is structurally no more complicated than the axisymmetric case [6-8].

In this paper we present the full structure, using Cartesian coordinates, of the autocorrelation and cross-correlation tensors associated with the solenoidal velocity and magnetic fluctuations in homogeneous turbulence. This provides complete information concerning the structure of all second- 
order moments (spatial correlation functions and corresponding spectra) for locally homogeneous and incompressible MHD turbulence, a model often found appropriate, for example, for the solar wind. Our aim is to remain clear and physically motivated, while employing reasonably rigorous derivations. Thus we do not treat nondifferentiable fields and other pathological cases. We also do not attempt to generalize to non-Cartesian coordinates. Along the way we find it useful to discuss and clarify several misleading or erroneous statements in the literature $[1,8,9,12]$. Fortunately, these inaccurate statements have remained in abstract terms and to our knowledge have not adversely influenced specific dynamical models or interpretation of data. We intend that the present, more complete, discussion will be useful in future models and the analysis of nonsymmetric homogeneous turbulence in a variety of applications.

\section{DEFINITIONS AND NOTATION}

We work with the zero-mean proper and pseudovector solenoidal fields, respectively denoted $\mathbf{v}(\mathbf{x})$ and $\mathbf{b}(\mathbf{x})$, with $\mathbf{x}$ the (Cartesian) position vector relative to some fixed origin. These quantities have obvious interpretations as the fluctuating parts of the velocity and magnetic fields in an incompressible MHD fluid (here $\mathbf{b}$ is in Alfvén speed units, for which the laboratory field is scaled by the factor $1 / \sqrt{4 \pi \rho}$, with $\rho$ the uniform mass density). In the final section we also summarize our results in terms of correlations between the Elsässer variables $\mathbf{z}^{ \pm}=\mathbf{v} \pm \mathbf{b}$, which are popular in solar wind transport theory, for example $[19,20]$.

Consider the definitions of the correlation and crosscorrelation tensors

$$
\begin{gathered}
R_{i j}^{v}(\mathbf{r})=\left\langle v_{i}(\mathbf{x}) v_{j}(\mathbf{x}+\mathbf{r})\right\rangle=\left\langle v_{i} v_{j}^{\prime}\right\rangle, \\
R_{i j}^{b}(\mathbf{r})=\left\langle b_{i} b_{j}^{\prime}\right\rangle, \\
R_{i j}^{v b}(\mathbf{r})=\left\langle v_{i} b_{j}^{\prime}\right\rangle, \\
R_{i j}^{ \pm}(\mathbf{r})=\frac{1}{2}\left\langle v_{i} b_{j}^{\prime} \pm b_{i} v_{j}^{\prime}\right\rangle .
\end{gathered}
$$

A prime denotes evaluation of the field at the displaced position $\mathbf{x}^{\prime}=\mathbf{x}+\mathbf{r}$ and the angular brackets denote an appropriately defined ensemble average, usually taken to be equivalent to long-time averaging (see, e.g., [4]). Under the assumption of homogeneity such correlation tensors are invariant with respect to a change of coordinate origin and thus depend only on the relative separation $\mathbf{r}$ [21].

We refer to $R^{v}, R^{b}$, and $R^{ \pm}$as the four "primary" tensors, with $R^{v b}$ used mainly as an intermediate form useful for obtaining the symmetrized versions $R^{ \pm}$. Unless otherwise stated, the absence of a $v, b,+$, or - superscript indicates that the equation applies equally well to all four primary forms. A subscript or superscript $v / b$ indicates the relation applies to both the $v$ and $b$ autocorrelation tensors and is not to be confused with the superscript $v b$ of Eq. (3) for example. Also, both the indexed and unindexed forms will be used to denote the same tensor, e.g., $R \equiv\left\{R_{i j} \mid i, j=1,2,3\right\}$ denotes the full tensor. The summation convention on repeated indices is in effect unless otherwise stated.
TABLE I. Some basic properties of the four " primary" spectral tensors. As far as the properties listed here are concerned, the $v / b$ tensors behave in the same fashion as the " + ", ones. See the text for details and the appropriate forms in $x$ space.

\begin{tabular}{lcc}
\hline \hline Property & Full tensor & Symmetrized components \\
\hline homogeneity & $S_{i j}^{ \pm}(\mathbf{k})= \pm S_{j i}^{ \pm}(-\mathbf{k})$ & $I_{i j}^{ \pm}(\mathbf{k})= \pm I_{i j}^{ \pm}(-\mathbf{k})$ \\
& & $J_{i j}^{ \pm}(\mathbf{k})=\mp J_{i j}^{ \pm}(-\mathbf{k})$ \\
solenoidality & $k_{i} S_{i j}^{ \pm}=k_{j} S_{i j}^{ \pm}=0$ & $k_{i} I_{i j}^{ \pm}=k_{j} I_{i j}^{ \pm}=0$ \\
& & $k_{i} J_{i j}^{ \pm}=k_{j} J_{i j}^{ \pm}=0$ \\
\hline \hline
\end{tabular}

As is well known, a general second-rank tensor $R$ may always be written as the sum of an index-symmetric part $I$ and an index-antisymmetric part $J$ where $I_{i j}(\mathbf{r})=\left[R_{i j}(\mathbf{r})\right.$ $\left.+R_{j i}(\mathbf{r})\right] / 2$ and $J_{i j}=\left(R_{i j}-R_{j i}\right) / 2$. Note that if $\mathbf{v}$ and $\mathbf{b}$ represent the usual MHD fields, then $R_{i j}^{-}(0)=\left\langle v_{i} b_{j}-b_{i} v_{j}\right\rangle$ is related to the average electromotive force (emf), which apart from a sign difference is the ensemble-averaged MHD induced electric field $\mathcal{E}=-\langle\mathbf{v} \times \mathbf{b}\rangle$. This connection is elaborated on in various sections.

The Fourier representation of the correlation tensors is also of importance. In general, we denote the Fourier transform of $f(\mathbf{r})$ by $f(\mathbf{k})$, e.g., $\mathbf{v}(\mathbf{k})=\int \mathbf{v}(\mathbf{r}) e^{i \mathbf{k} \cdot \mathbf{r}} d^{3} r /(2 \pi)^{3}$. However, it is customary to denote the Fourier transforms of the $R(\mathbf{r})$ by $S(\mathbf{k})$, and we follow this practice. Thus, for example, $I_{i j}^{ \pm}(\mathbf{r})$ and $I_{i j}^{ \pm}(\mathbf{k})$ are Fourier transforms of each other, as are $R_{i j}^{v}(\mathbf{r})$ and $S_{i j}^{v}(\mathbf{k})$. The definitions $k=|\mathbf{k}|$ and $\hat{\mathbf{k}}=\mathbf{k} / k$ are also employed, and in general a caret will be used to signify unit vectors.

Some of the important properties of the $S$ 's, I's, and $J$ 's are summarized in Tables I and II, where the anomalous behavior of the "minus" tensors is evident. Further elementary definitions and results are contained in Appendix A.

\section{UNDERLYING THEORY AND PROPERTIES}

\section{A. Theory for construction of the tensors}

Forms for the correlation functions can be constructed in several different ways. One method employs the theory of isotropic tensors, developed by Robertson [1] and Chandrasekhar $[7,9,10]$. In outline their procedure is as follows.

(i) List all possible dyadic "construction elements" that can be formed from combinations of the fundamental vectors (and tensors) in the problem and the two isotropic tensors $\delta_{i j}$ and $\epsilon_{i j m}$ (e.g., $r_{i} r_{j}, \epsilon_{i j \alpha} r_{\alpha}$ ). Each of these tensor forms corresponds to a possible scalar that can be extracted from the turbulence field by contracting the correlation tensor with a pair of vectors $\mathbf{a}, \mathbf{c}$, such as $\mathbf{a} \cdot \mathbf{c}$ or $\mathbf{a} \cdot(\mathbf{r} \times \mathbf{c})$.

(ii) Associate a multiplicative function with each construction element, representing information specific to the turbulence ensemble. These scalar generating functions depend only on the invariant scalars formed from contractions of the fundamental vectors of the problem with the available isotropic tensors, for example, $A\left(r^{2}, r_{z}\right)$, where $r^{2}=\mathbf{r} \cdot \mathbf{r}$, $r_{z}=\mathbf{r} \cdot \hat{\mathbf{B}}_{0}$, and $\mathbf{B}_{0}$ is some preferred direction.

(iii) Form the sum of all such terms.

(iv) Impose appropriate constraints on the resulting form (e.g., solenoidality and homogeneity). 
TABLE II. Additional properties of the index-symmetric $(I)$ and antisymmetric $(J)$ components of the "primary" spectral tensors. Note the anomalous behavior of the minus tensors. In $x$ space, columns 2, 4, and 5 still apply, but all quantities are of course purely real.

\begin{tabular}{lccccc}
\hline \hline Tensor & $\begin{array}{c}\text { Tensor } \\
\text { character }\end{array}$ & $\begin{array}{c}\text { Real or } \\
\text { imaginary }\end{array}$ & $\begin{array}{c}\text { Index } \\
\text { symmetry }\end{array}$ & $\begin{array}{c}\mathbf{k} \\
\text { parity }\end{array}$ & $\begin{array}{c}\text { Hermitian } \\
\text { nature }\end{array}$ \\
\hline$I^{v}(\mathbf{k}), I^{b}(\mathbf{k})$ & true & $\operatorname{Re}$ & symmetric & even & Hermitian \\
$I^{+}(\mathbf{k})$ & pseudo & $\mathrm{Re}$ & $\begin{array}{c}\text { symmetric } \\
\text { symmetric }\end{array}$ & odd & $\begin{array}{c}\text { Hermitian } \\
\text { anti-Hermitian }\end{array}$ \\
$I^{-}(\mathbf{k})$ & pseudo & $\mathrm{Im}$ & antisymmetric & odd & Hermitian \\
$J^{v}(\mathbf{k}), J^{b}(\mathbf{k})$ & true & $\mathrm{Im}$ & antisymmetric & odd & Hermitian \\
$J^{+}(\mathbf{k})$ & pseudo & $\mathrm{Im}$ & antisymmetric & even & anti-Hermitian \\
$J^{-}(\mathbf{k})$ & pseudo & $\mathrm{Re}$ & & & \\
\hline \hline
\end{tabular}

The final step yields relationships among the various terms in the general expression and allows the minimum number of scalar generating functions to be determined. One then arrives at the minimal specification of the most general correlation tensor allowed by the set of assumed symmetries.

In Sec. IV we shall proceed via a distinct route, wherein the solenoidal constraint is built in at an early stage, versus being enforced as a final step. This reordering of the standard approach is more convenient when preferred directions are permitted, i.e., for any symmetry more complex than isotropy. For the case of axisymmetric turbulence, Chandrasekhar [7] introduced a related method for enforcement of the solenoidal condition, based on taking the curl of appropriate potential correlations to arrive at the velocity correlation tensor.

Two approaches to identifying the generating functions will be presented below. The first makes use of the vector potentials for $\mathbf{v}$ and $\mathbf{b}$ and avoids using any arbitrary preferred direction(s). By expressing the spectral tensor as the correlation between the curls of these potentials, we are able to examine the number and type of scalar degrees of freedom appearing in the most general second-rank correlation tensors involving $\mathbf{v}$ and $\mathbf{b}$. We conclude, in accordance with the argument of Orszag [22], that four such scalars exist in general. This procedure justifies the subsequent use of a k-dependent coordinate system, involving an arbitrary reference direction and scalar potentials (i.e., the poloidal and toroidal potentials; see Appendix B), to economically develop explicit representations of the basic tensor forms. Although the latter is conceptually less elegant, it nonetheless proves to be a powerful approach. Before considering either of these methods, however, we discuss the importance of pseudoscalar and pseudotensor contributions to the correlation tensors.

\section{B. Scalars vs pseudoscalars}

In Robertson's seminal discussion of second-rank isotropic autocorrelation tensors [1], contributions are divided according to whether their associated scalars, obtained after contraction with arbitrary vectors $\mathbf{a}$ and $\mathbf{c}$, are of type (1) (inner products like $\mathbf{a} \cdot \mathbf{c}$ ) or type (2) [triple products like $\mathbf{a} \cdot(\mathbf{r} \times \mathbf{c})]$. If $A, B, C$ are scalar functions of the separation distance $|\mathbf{r}|$, tensor forms associated with type (1) scalars are $A r_{i} r_{j}$ and $B \delta_{i j}$, while the isotropic form connected with type (2) scalars is $C \epsilon_{i j \alpha} r_{\alpha}$. In order to impose the assump- tion that the measured scalar correlations are invariant under the extended (proper and improper) rotation group, Robertson argues that "such a scalar invariant under this extended group can be expressed in terms of scalars of type (1) alone, for those of type (2) suffer a change of sign under reflexion ...', On this basis he in effect chooses $C=0$ and arrives at the correct [type (1)] form for mirror-symmetric isotropic correlations. However, upon considering the non-mirrorsymmetric case more carefully, one sees that Robertson's reasoning is imprecise, however correct his conclusions may have been.

The deficiency in this reasoning is seen even in the simplest case of isotropic helical turbulence, in which the indexantisymmetric type (2) correlation takes the form $\epsilon_{i j \alpha} \partial \Phi(r) / \partial r_{\alpha}[13,23]$, where one can show that the helicity generating function satisfies $\nabla^{2} \Phi(0)=-\langle\mathbf{u} \cdot \boldsymbol{\omega}\rangle / 2$. The quantity $\mathbf{u} \cdot \boldsymbol{\omega}$ is a pseudoscalar, reversing sign under inversion, and therefore it is clear that $\Phi$ is also a pseudoscalar. Consequently the above-mentioned type (2) indexantisymmetric form does not reverse sign under inversion and scalars derived from it (e.g., $\mathbf{a} \cdot \mathbf{c} \times \nabla \Phi)$ are proper scalars and not pseudoscalars. In Robertson's notation, this case corresponds to inclusion of a type (2) contribution of the form $\epsilon_{i j \alpha} r_{\alpha}$; this second-rank pseudotensor is multiplied by a pseudoscalar $C$. The product does not reverse sign under inversions, even though nonmirror reflection invariant correlations associated with the helicity $\langle\mathbf{u} \cdot \boldsymbol{\omega}\rangle$ are present.

This conclusion is beyond the scope of Robertson's considerations because he assumed no helical correlations. For the index-symmetric autocorrelation tensors he considers, Robertson's argument leads to no errors. However, his reasoning does not carry over to the case of type (2) contributions, in which both pseudotensor forms and pseudoscalar generating functions can appear.

Several other errors related to this point exist in the literature, especially with regard to the index-antisymmetric helicitylike correlations. In connection with isotropic but nonmirror symmetric MHD correlations, Chandrasekhar [9] concluded incorrectly [in his Eq. (18)] that the $\left\langle v_{i} v_{j}^{\prime}\right\rangle$ and $\left\langle b_{i} b_{j}^{\prime}\right\rangle$ are necessarily index symmetric and that the cross correlation $\left\langle v_{i} b_{j}^{\prime}\right\rangle$ is purely index antisymmetric and of the form $C \epsilon_{i j \alpha} r_{\alpha}$. The former statement is too restrictive and disallows helical correlation, while the second is clearly incorrect, as can be seen by the fact that $\left\langle v_{i} v_{j}^{\prime}\right\rangle$ and $\left\langle v_{i} b_{j}^{\prime}\right\rangle$ must be structurally similar as the cross helicity $\mathbf{v} \cdot \mathbf{b}$ be- 
comes large. In fact, under Chandrasekhar's assertion, $\left\langle v_{i} b_{i}^{\prime}\right\rangle \equiv 0$ and the so-called Alfvénic fluctuations that are observed in the solar wind (see, e.g., [24]) would be impossible. Both of Chandrasekhar's oversights are corrected by including appropriate pseudoscalar functions and associated tensor or pseudotensor forms. For example, $\left\langle v_{i} b_{j}^{\prime}\right\rangle$ should include a symmetric part and $\left\langle v_{i} v_{j}^{\prime}\right\rangle$ an antisymmetric part, as will be discussed in detail below.

The paper by Matthaeus and Smith [8] also contains an error. They stated that " any homogeneous correlation matrix $R$ of the form $R_{i j}(\mathbf{r})=\left\langle b_{i}(\mathbf{x}) b_{j}(\mathbf{x}+\mathbf{r})\right\rangle$ consists of the sum of a symmetric proper tensor and an antisymmetric pseudotensor, independent of the vector or pseudovector nature of b," which they refer to as Theorem A. We can now see that this theorem is incorrect.

A correct statement that replaces the Matthaeus-Smith assertion (without invalidating their main conclusions) and also serves to clarify the situations discussed by Robertson and Chandrasekhar is the following

Theorem $A A$ : $R^{v}$ and $R^{b}$ are proper (true) tensors, while $R^{ \pm}$are pseudotensors.

To prove the theorem for $R^{b}$ we proceed as follows.

(i) Choose a particular coordinate system basis and define $R^{b}$ (r) with respect to it, using Eq. (2).

(ii) Make an orthogonal change of basis via the transformation matrix $M$, where $\operatorname{det}(M)= \pm 1$. In the new coordinate system we have, for example, $\widetilde{\mathbf{x}}=\mathbf{M x}, \widetilde{\mathbf{v}}=\mathbf{M v}$, and $\widetilde{\mathbf{b}}=\operatorname{det}(\mathrm{M}) \mathrm{Mb}$ and the correlation function is defined by $\widetilde{R}_{\alpha \beta}^{b}(\widetilde{\mathbf{r}})=\left\langle\widetilde{b}_{\alpha}(\widetilde{\mathbf{x}}) \widetilde{b}_{\beta}(\widetilde{\mathbf{x}}+\widetilde{\mathbf{r}})\right\rangle$.

(iii) It is then straightforward to show that $\widetilde{R}_{\alpha \beta}^{b}(\widetilde{\mathbf{r}})=\mathrm{M}_{\alpha i} \mathrm{M}_{\beta j} R_{i j}^{b}(\mathrm{Mr})$ and thus $R^{b}$ is a true tensor.

The proofs for $R^{v}$ and $R^{ \pm}$are analogous. Hence all additive elements of a given correlation tensor have the same overall true or pseudo nature. While such proofs may seem obvious, apparently they have not been published previously in the context of MHD turbulence theory. These results have important consequences for correlation tensors. For example, for the $v / b$ tensors each additive component of $R$ can only be either a true tensor multiplied by a true scalar function or a pseudotensor multiplied by a pseudoscalar function.

In some of the earlier literature it was erroneously assumed (tacitly in some cases) that the scalar functions multiplying the "bare" tensor forms must always be proper scalars. Above we argued that $\Phi(\mathbf{r})$ is a pseudoscalar because when evaluated at the origin it is a one-point pseudoscalar correlation. Indeed, it is instructive to demonstrate that the antisymmetric tensor that appears in several of the autocorrelation and cross-correlation tensors described below is always connected with nonzero values of various one-point pseudoscalar correlations.

To be definite consider the antisymmetric part of the autocorrelation $\left\langle v_{i} v_{j}^{\prime}\right\rangle$, which can always be written as $\epsilon_{i j \alpha} \partial \Phi(\mathbf{r}) / \partial r_{\alpha}[15,25]$, which applies to all homogeneous velocity fluctuations and generalizes the isotropic form mentioned above. The antisymmetric part of the correlation tensor must be an odd function of $\mathbf{r}$ and thus $\Phi$ is even. Assuming well-behaved correlation functions, we can express the generating function as a power series $\Phi(\mathbf{r})$ $=a+b_{i j} r_{i} r_{j}+c_{i j l m} r_{i} r_{j} r_{l} r_{m}+\cdots$. Matthaeus and Smith [15] showed that $\langle\mathbf{v} \cdot \boldsymbol{\psi}\rangle=2 a$, where $\mathbf{v}=\nabla \times \boldsymbol{\psi}$. Similarly, as was found for the isotropic case, we have $\langle\mathbf{u} \cdot \boldsymbol{\omega}\rangle=$ $-2 \nabla^{2} \Phi(0)=-4 b_{i i}$. It is not difficult to go further and derive an explicit form for the matrix

$$
b_{i j}=\frac{1}{4}\left\langle v_{\alpha} \nabla_{j} v_{\beta}\right\rangle \epsilon_{i \alpha \beta},
$$

which can be verified by direct construction. Similarly, one can find explicit forms for higher-order coefficients, such as

$$
c_{i j l m}=\frac{1}{2 \times 4 !}\left\langle v_{\alpha} \nabla_{j} \nabla_{l} \nabla_{m} v_{\beta}\right\rangle \epsilon_{i \alpha \beta} .
$$

Note that all of the matrix coefficients appearing in the expansion of $\Phi$ can be assumed to be symmetric under interchange of any pair of indices. The generalization to remaining orders is clear. Each case involves one $\epsilon_{i \alpha \beta}$ and an odd number of derivatives acting on the second argument of the correlation tensor, which is to be evaluated at zero separation and contracted appropriately. Each of these coefficients is manifestly a pseudoscalar derived from one-point correlations. If all such pseudoscalars vanish, so will the helicity generating function $\Phi$, along with the entire antisymmetric part of the correlation tensor.

In light of this example and theorem AA, a refinement of Robertson's statement is as follows. An autocorrelation tensor is a proper tensor. Type (1) tensor forms appear multiplied by proper scalar functions. Type (2) forms are multiplied by pseudoscalars. In the latter case the presence of a nonzero pseudoscalar function is connected with the nonvanishing of at least some of the natural pseudoscalars that can be formed from one-point correlations of the basic fields.

Note that each one-point pseudoscalar correlation appearing in the above expansion is directly related to (one of) the generalized helicities (e.g., $\langle\boldsymbol{\psi} \cdot \mathbf{v}\rangle,\langle\mathbf{v} \cdot \boldsymbol{\omega}\rangle,\langle\boldsymbol{\omega} \cdot \nabla \times \boldsymbol{\omega}\rangle$, etc.) and these are interpretable [26,27] as linkages and twists of the appropriate field. Statements analogous to the above hold for a pseudotensor cross correlation involving a vector and a pseudovector quantity.

\section{EXPLICIT TENSOR FORMS: VECTOR POTENTIALS}

Since both $\mathbf{v}$ and $\mathbf{b}$ are solenoidal, it is convenient to introduce potentials $\boldsymbol{\psi}$ and $\mathbf{A}$ such that

$$
\mathbf{v}(\mathbf{x})=\nabla \times \boldsymbol{\psi}(\mathbf{x}), \quad \mathbf{b}(\mathbf{x})=\nabla \times \mathbf{A}(\mathbf{x}),
$$

where the vector potentials are only unique up to the addition of the gradient of an arbitrary function of position (gauge freedom). In Fourier space these relations become $\mathbf{v}(\mathbf{k})=i \mathbf{k} \times \boldsymbol{\psi}(\mathbf{k})$ and $\mathbf{b}(\mathbf{k})=i \mathbf{k} \times \mathbf{A}(\mathbf{k})$. It follows that $\boldsymbol{\omega}(\mathbf{x})=\nabla \times \mathbf{v}(\mathbf{x})=-\nabla^{2} \boldsymbol{\psi}+\nabla \nabla \cdot \boldsymbol{\psi} \quad$ and $\quad \boldsymbol{\omega}(\mathbf{k})=i \mathbf{k} \times \mathbf{v}(\mathbf{k})$ $=k^{2} \boldsymbol{\psi}-\mathbf{k}(\mathbf{k} \cdot \boldsymbol{\psi})$, with similar relationships holding for $\mathbf{j}=\nabla \times \mathbf{b}$. When $\mathbf{v}$ and $\mathbf{b}$ are the usual MHD fields, $\boldsymbol{\psi}$ is the velocity stream function, $\boldsymbol{\omega}$ the vorticity, and $\mathbf{j}$ the electric current density (in appropriate units).

Making the appropriate substitutions in the Fourier transform of Eq. (3) we obtain 


$$
\begin{aligned}
S_{i j}^{v b}(\mathbf{k})= & \left\langle v_{i}^{*} b_{j}\right\rangle \\
= & k^{2}\left[\delta_{i j}-\frac{k_{i} k_{j}}{k^{2}}\right]\left\langle\boldsymbol{\psi}^{*} \cdot \mathbf{A}\right\rangle+\left\langle\mathbf{k} \cdot \boldsymbol{\psi}^{*}\left\{A_{i} k_{j}-\delta_{i j} \mathbf{k} \cdot \mathbf{A}\right\}\right\rangle \\
& +\left\langle\psi_{j}^{*}\left\{k_{i} \mathbf{k} \cdot \mathbf{A}-k^{2} A_{i}\right\}\right\rangle .
\end{aligned}
$$

Specializing to the Coulomb gauge for both $\boldsymbol{\psi}$ and $\mathbf{A}$, i.e., $\mathbf{k} \cdot \boldsymbol{\psi}=0=\mathbf{k} \cdot \mathbf{A}$, yields

$$
S_{i j}^{v b}(\mathbf{k})=k^{2}\left[\delta_{i j}-\frac{k_{i} k_{j}}{k^{2}}\right]\left\langle\boldsymbol{\psi}^{*} \cdot \mathbf{A}\right\rangle-k^{2}\left\langle\psi_{j}^{*} A_{i}\right\rangle .
$$

The forms for the autocorrelations of $\mathbf{v}$ and $\mathbf{b}$ follow by letting $\mathbf{A} \mapsto \boldsymbol{\psi}$ and $\boldsymbol{\psi} \mapsto \mathbf{A}$, respectively.

Continuing to work in the Coulomb gauge, we may form the " \pm " tensors [cf. Eq. (4)]

$$
\begin{aligned}
S_{i j}^{ \pm}(\mathbf{k}) & =S_{i j}^{v b} \pm\left(S_{j i}^{v b}\right)^{*} \\
& =k^{2}\left[\delta_{i j}-\frac{k_{i} k_{j}}{k^{2}}\right]\left\langle\boldsymbol{\psi}^{*} \cdot \mathbf{A} \pm \text { H.c. }\right\rangle \mp k^{2}\left\langle\psi_{i} A_{j}^{*} \pm \text { H.c. }\right\rangle,
\end{aligned}
$$

where H.c. indicates the Hermitian conjugate (transpose of the complex conjugate). Alternative representations of $S^{ \pm}$ can be formed using relations such as $\boldsymbol{\omega}=k^{2} \boldsymbol{\psi}$.

While this approach can be useful, it does not straightforwardly lend itself to extracting explicit forms for the scalar generating functions. However, Eqs. (9) and (10) can be used to prove, in a coordinate system independent way, that for each $S$ (or $R$ ) there are precisely four independent scalar generating functions (Orszag [22] has given a distinct, essentially geometric, proof). The proof follows.

Consider, as an example, $S^{+}(\mathbf{k})$. Equation (10) shows that the independent elements of $S^{+}$are completely determined by those of the tensor $\Pi_{i j}=\left\langle\psi_{i} A_{j}^{*}+\psi_{j}^{*} A_{i}\right\rangle$. This is clearly a Hermitian matrix and so is fully determined by at most nine independent real numbers. However, some of the degrees of freedom in $\Pi$ are associated with gauge freedoms that do not influence the correlation tensor. Choosing the Coulomb gauge for both $\boldsymbol{\psi}$ and $\mathbf{A}$, for example, implies that $k_{i} \Pi_{i j}=\Pi_{i j} k_{j}=0$ and further constrains the number of independent elements. In this gauge, $\Pi$ can be written

$$
\left(\begin{array}{ccc}
d_{1} & c+i h & 0 \\
c-i h & d_{2} & 0 \\
0 & 0 & 0
\end{array}\right)
$$

where the nonzero $2 \times 2$ subspace is orthogonal to $\mathbf{k}$ and $d_{1}, d_{2}, c$, and $h$ are real. Thus $S^{+}(\mathbf{k})$ has four independent scalar elements. Montgomery and Turner [28] considered a similar decomposition without reference to the potentials; see also [29]. The same argument can be applied to $S^{v / b}$, which is also Hermitian, and an analogous argument used for the anti-Hermitian $S^{-}(\mathbf{k})$. It is noteworthy that the above argument proceeds without choosing a coordinate system (or an arbitrary reference direction) for $S$.

When $h$ is zero (clearly connected to the absence of helicity), Eq. (11) can be diagonalized by a real rotation, allow- ing $c$ to be "hidden" in the diagonal terms. However, in general the diagonalization cannot be performed simultaneously for all $\mathbf{k}$, so that $c$ remains as a degree of freedom. Note that the turbulence is isotropic if and only if, for all $\mathbf{k}, c=0$ and $d_{1}=d_{2}$. Similarly, when $h \neq 0$ a unitary transformation can eliminate the degrees of freedom associated with both $h$ and $c$, for a given $\mathbf{k}$. In general, such a procedure does not eliminate degrees of freedom in $S$ since $h$ and $c$ are functions of $\mathbf{k}$ and hence a distinct unitary transformation is required for each $\mathbf{k}$ mode.

\section{EXPLICIT TENSOR FORMS: SCALAR POTENTIALS}

\section{A. Real space potentials}

Having shown that four scalar functions are necessary to specify each of the correlation functions (1)-(4), we derive explicit forms for the correlation tensors. This is facilitated by employing representations based on an arbitrary reference direction.

As reviewed in Appendix B, a solenoidal vector field can always be decomposed into poloidal and toroidal contributions. We use the $x$-space forms

$$
\begin{aligned}
& \mathbf{v}=-\nabla \times\left(\mathbf{e} \times \nabla P_{v}\right)-\mathbf{e} \times \nabla T_{v}, \\
& \mathbf{b}=-\nabla \times\left(\mathbf{e} \times \nabla P_{b}\right)-\mathbf{e} \times \nabla T_{b},
\end{aligned}
$$

where the $P$ 's are the poloidal potentials and the $T$ 's the toroidal ones. For convenience we refer to the scalar functions $P$ and $T$ as the poloidal and toroidal potentials of the vector field, although strictly speaking these terms apply only to the associated vector components of the field. Since $\mathbf{v}$ is a true vector, $P_{v}$ must be a true scalar and $T_{v}$ a pseudoscalar; similarly, $P_{b}$ is a pseudoscalar and $T_{b}$ a true scalar. This information will be of use for an alternative proof of theorem AA.

Note that $\mathbf{e}$ is an arbitrary reference direction that has no intrinsic connection with possible preferred directions existing in the turbulence (e.g., a rotation axis or uniform magnetic field). Nonetheless, in some circumstances it is useful to consider $\mathbf{e}$ to coincide with such a preferred direction (cf. Sec. VII).

We begin with $R_{i j}^{v}(\mathbf{r})$. From Eq. (12) it follows that (since no confusion should result, we temporarily drop the $v$ subscripts on $P_{v}$ and $T_{v}$ )

$$
\begin{gathered}
v_{i}(\mathbf{x})=-e_{i} \frac{\partial^{2} P(\mathbf{x})}{\partial x_{\alpha} \partial x_{\alpha}}+e_{\alpha} \frac{\partial^{2} P(\mathbf{x})}{\partial x_{i} \partial x_{\alpha}}-\epsilon_{i \alpha \beta} e_{\alpha} \frac{\partial T(\mathbf{x})}{\partial x_{\beta}}, \\
v_{j}(\mathbf{x}+\mathbf{r})=-e_{j} \frac{\partial^{2} P(\mathbf{x}+\mathbf{r})}{\partial r_{\mu} \partial r_{\mu}}+e_{\mu} \frac{\partial^{2} P(\mathbf{x}+\mathbf{r})}{\partial r_{j} \partial r_{\mu}} \\
-\epsilon_{j \mu \nu} e_{\mu} \frac{\partial T(\mathbf{x}+\mathbf{r})}{\partial r_{\nu}} .
\end{gathered}
$$

After multiplying these together and ensemble averaging we obtain an intermediate form for $R_{i j}^{v}$ that involves the functions $A(\mathbf{r})=\left\langle P P^{\prime}\right\rangle, B=\left\langle T T^{\prime}\right\rangle, C_{1}=\left\langle P T^{\prime}\right\rangle, \quad$ and $C_{2}(\mathbf{r})$ $=\left\langle P^{\prime} T\right\rangle=C_{1}(-\mathbf{r})$. For simplicity we suppress the $v$ subscripts that should be attached to these functions and their descendants. To collect terms of like symmetry let 
$2 D=C_{1}+C_{2}$ and $2 C=C_{1}-C_{2}$. Homogeneity requires that $A, B$, and $D$ are even under the coordinate inversion $\mathbf{r} \mapsto-\mathbf{r}$, while $\mathrm{C}$ is odd. We then eventually obtain

$$
\begin{aligned}
R_{i j}^{v}(\mathbf{r})= & {\left[\partial_{i j}^{2}(\mathbf{e} \cdot \nabla)^{2}-\left(e_{i} \partial_{j}+e_{j} \partial_{i}\right)(\mathbf{e} \cdot \nabla) \nabla^{2}+e_{i} e_{j} \nabla^{2} \nabla^{2}\right] A } \\
& -\left\{\delta_{i j}\left[\nabla^{2}-(\mathbf{e} \cdot \nabla)^{2}\right]+\partial_{i j}^{2}-\left(e_{i} \partial_{j}+e_{j} \partial_{i}\right)(\mathbf{e} \cdot \nabla)\right. \\
& \left.+e_{i} e_{j} \nabla^{2}\right\} B+\left[\left(e_{i} \epsilon_{j \mu \nu}+e_{j} \epsilon_{i \mu \nu}\right) e_{\mu} \partial_{\nu} \nabla^{2}-\left(\epsilon_{i \mu \nu} \partial_{j}\right.\right. \\
& \left.\left.+\epsilon_{j \mu \nu} \partial_{i}\right) e_{\mu} \partial_{\nu}(\mathbf{e} \cdot \nabla)\right] C-\epsilon_{i j \alpha} \partial_{\alpha}\left[\nabla^{2}-(\mathbf{e} \cdot \nabla)^{2}\right] D
\end{aligned}
$$

where $\partial_{i j}^{2}=\partial^{2} / \partial r_{i} \partial r_{j}$, etc. Clearly, only the terms involving $D$ are index antisymmetric, while the others are strictly index symmetric. It is convenient to absorb factors like $\mathbf{e} \cdot \nabla$ into the scalar functions. Thus let

$$
\begin{gathered}
\nabla^{2} F=B+\nabla^{2} A, \\
\nabla^{2} E=-\left[\nabla^{2}-(\mathbf{e} \cdot \nabla)^{2}\right] B, \\
H=-\left[\nabla^{2}-(\mathbf{e} \cdot \nabla)^{2}\right] D .
\end{gathered}
$$

The evenness of $A, B$, and $D$ under coordinate inversion, implies that $E, F$, and $H$ are also even functions of $\mathbf{r}$. It can also be shown that $(\mathbf{e} \cdot \nabla)^{2} A+B=(\mathbf{e} \cdot \nabla)^{2} F-E$, provided that all the generating functions have vanishing spatial mean value. This relation is useful in determining the final form

$$
\begin{aligned}
R_{i j}^{v}(\mathbf{r})= & {\left[\delta_{i j} \nabla^{2}-\partial_{i j}^{2}\right] E_{v}-\left[\left(e_{i} \partial_{j}+e_{j} \partial_{i}\right)(\mathbf{e} \cdot \nabla) \nabla^{2}+e_{i} e_{j} \nabla^{2} \nabla^{2}\right.} \\
& \left.+\partial_{i j}^{2}(\mathbf{e} \cdot \nabla)^{2}\right] F_{v}+\left(\delta_{i \alpha} \epsilon_{j \mu \nu}+\delta_{j \alpha} \epsilon_{i \mu \nu}\right) e_{\mu} \partial_{\nu}\left[e_{\alpha} \nabla^{2}\right. \\
& \left.-\partial_{\alpha}(\mathbf{e} \cdot \nabla)\right] C_{v}+\epsilon_{i j \alpha} \partial_{\alpha} H_{v},
\end{aligned}
$$

where we have reinstated the $v$ subscripts of the scalar functions.

Thus, for incompressible homogeneous turbulence the index-symmetric part of the velocity correlation tensor can be generated from three independent scalar functions, while the index-antisymmetric part depends on a single such function $H_{v}$. As mentioned above, this fourfold generation of the correlation tensors will be shown to apply for both the autocorrelation and the cross-correlation tensors considered here. The parity of the scalar functions has also been explicitly obtained: $E_{v}, F_{v}$, and $H_{v}$ are even, whereas $C_{v}$ is odd. Clearly, an exactly analogous derivation, and thus final form, holds for $R_{i j}^{b}$, the true or pseudovector nature of the underlying field being immaterial for an autocorrelation. The spectral tensors are easily obtained via application of the Fourier transform.

Note that the result is independent of the true or pseudovector nature of $\mathbf{e}$ since components of $\mathbf{e}$ only appear in product pairs. This will be of importance when we consider homogeneous turbulence with an externally enforced preferred direction, such as that due to a uniform magnetic field (see Sec. VII C).

Equation (17) is structurally equivalent to the result given as Eq. (18) in Ref. [8]. This reveals a formal coincidence of the general correlation tensors with those of axisymmetric homogeneous turbulence if $\mathbf{e}$ is specialized to be an axis of symmetry and the scalars, in general functions of $\mathbf{r}$, are cho- sen to be independent of rotations about that axis. This correspondence is discussed further in Sec. VII, along with other special cases. For isotropic turbulence, however, there can be no dependence on any particular direction and thus no dependence on e. Therefore, we must have $F_{v}=C_{v}=0$, and the well-known result is recovered if $E_{v}$ and $H_{v}$ are functions of $|\mathbf{r}|$.

\section{B. Fourier space potentials}

To complement the derivation of the $x$-space forms of the $v, b$ tensors, we now outline the derivation of the $k$-space forms for the \pm tensors. As discussed in Appendix B, it is convenient to choose the two scalar potentials to have the same dimensions. Using the $k$-space forms of Eqs. (12) and (13), with the extra factors of $1 / k$ inserted, we obtain

$$
v_{i}^{*}(\mathbf{k})=\left[k^{2} e_{i}-\mathbf{e} \cdot \mathbf{k} k_{i}\right] \frac{P_{v}^{*}(\mathbf{k})}{k}+i(\mathbf{e} \times \mathbf{k})_{i} T_{v}^{*}(\mathbf{k}),
$$

etc. The Fourier transform of Eq. (3) is $S_{i j}^{v b}$ $=\left\langle v_{i}(-\mathbf{k}) b_{j}(\mathbf{k})\right\rangle$. Substituting Eq. (18) and the analogous form for $b_{j}(\mathbf{k})$ into this and defining $A_{1}(\mathbf{k})=\left\langle P_{v}(-\mathbf{k}) P_{b}(\mathbf{k})\right\rangle=\left\langle P_{v}^{*} P_{b}\right\rangle, B_{1}(\mathbf{k})=\left\langle T_{v}^{*} T_{b}\right\rangle, C_{3}(\mathbf{k})$ $=\left\langle P_{v}^{*} T_{b}\right\rangle, C_{4}(\mathbf{k})=\left\langle T_{v}^{*} P_{b}\right\rangle$, and $C_{3}=D_{v b}+C_{v b}, C_{4}=D_{v b}$ $-C_{v b}, \quad E_{v b}=\left[k^{2}-(\mathbf{e} \cdot \mathbf{k})^{2}\right] B_{1}, \quad F_{v b}=B_{1}-A_{1}, H_{v b}=k[1$ $\left.-(\mathbf{e} \cdot \hat{\mathbf{k}})^{2}\right] D_{v b}$, and using the Chandrasekhar identities $[7,30]$ we find

$$
\begin{aligned}
S_{i j}^{v b}(\mathbf{k})= & {\left[\delta_{i j}-\frac{k_{i} k_{j}}{k^{2}}\right] E_{v b}+\left[\left[e_{i} k_{j}+e_{j} k_{i}\right](\mathbf{e} \cdot \mathbf{k})-e_{i} e_{j} k^{2}\right.} \\
& \left.-\frac{k_{i} k_{j}}{k^{2}}(\mathbf{e} \cdot \mathbf{k})^{2}\right] F_{v b}-i\left[\delta_{i \mu} \epsilon_{j \alpha \beta}+\delta_{j \mu} \epsilon_{i \alpha \beta}\right] \\
& \times e_{\alpha} k_{\beta}\left(e_{\mu} k^{2}-k_{\mu} \mathbf{e} \cdot \mathbf{k}\right) C_{v b}+i \epsilon_{i j \alpha} k_{\alpha} H_{v b} \cdot
\end{aligned}
$$

As was the case for the velocity and magnetic field autocorrelation tensors, the $E, F$, and $C$ terms are index symmetric and the $H$ term is antisymmetric. The part with indices associated with $C_{v b}$ can also be written $[\mathbf{k} \times(\mathbf{e} \times \mathbf{k})]_{i}(\mathbf{e} \times \mathbf{k})_{j}+[\mathbf{k} \times(\mathbf{e} \times \mathbf{k})]_{j}(\mathbf{e} \times \mathbf{k})_{i}, \quad$ showing that $C_{v b}$ does not contribute to the trace. Letting $P_{b} \rightarrow P_{v}$ and $T_{b} \rightarrow T_{v}$ recovers the result for the velocity autocorrelation; however, the result is not equal to the Fourier transform of Eq. (16) because we are working with $k$-space potentials that are dimensionally equal. Additionally, various factors of -1 are present, e.g., $-k^{2} E_{v b}(\mathbf{k}) \leftrightarrow E_{v b}(\mathbf{r})$.

Note that when the turbulence is isotropic equal amounts of energy must be associated with the poloidal and toroidal components since no real distinction between them then exists. This requires that $A_{1}=B_{1}$, and thus $F_{v b}=0$, providing additional proof that the $F$ scalar functions vanish for isotropic turbulence.

We now have everything we need to form the spectral versions of the \pm tensors. Referring to Eq. (19) we obtain 


$$
\begin{aligned}
S_{i j}^{ \pm}(\mathbf{k})= & S_{i j}^{v b} \pm\left(S_{j i}^{v b}\right) * \\
= & {\left[\delta_{i j}-\frac{k_{i} k_{j}}{k^{2}}\right] E^{ \pm}+\left[\left[e_{i} k_{j}+e_{j} k_{i}\right](\mathbf{e} \cdot \mathbf{k})-e_{i} e_{j} k^{2}\right.} \\
& \left.-\frac{k_{i} k_{j}}{k^{2}}(\mathbf{e} \cdot \mathbf{k})^{2}\right] F^{ \pm}-i\left[\delta_{i \mu} \epsilon_{j \alpha \beta}+\delta_{j \mu} \epsilon_{i \alpha \beta}\right] e_{\alpha} k_{\beta}\left(e_{\mu} k^{2}\right. \\
& \left.-k_{\mu} \mathbf{e} \cdot \mathbf{k}\right) C^{\mp}+i \epsilon_{i j \alpha} k_{\alpha} H^{ \pm},
\end{aligned}
$$

where $E^{ \pm}(\mathbf{k})=E_{v b}(\mathbf{k}) \pm E_{v b}(-\mathbf{k})$, etc., with the $+(-)$ form being explicitly even (odd) in k. An inverse Fourier transform yields the correlation tensors. Note that the + tensor involves $C^{-}$, not $C^{+}$, and vice versa.

So again the representation is one where three scalar functions are required for the symmetric terms and one for the antisymmetric portion. For $S^{+}$, these scalar functions have the same parity as their autocorrelation cousins, i.e., $E^{+}, F^{+}, H^{+}$are even and $C^{-}$odd under $\mathbf{k} \mapsto-\mathbf{k}$. The minus tensor, however, exhibits precisely complementary behavior with $E^{-}, F^{-}, H^{-}$being odd and $C^{+}$even. Thus, aside from the true or pseudo nature of the tensors (see Table II), $S_{i j}^{+}$is formally equivalent to $S_{i j}^{v}$ and $S_{i j}^{b}$, and similarly for their Fourier transforms. The equivalence means that many mathematical results that hold for any one of the three also hold for the other two. We subsequently refer to these tensors and their component scalar functions as "normal," while the minus tensor and its associated scalar functions are referred to as "anomalous."

\section{Further mathematical consequences}

Having derived the most general form of the four primary correlation tensors, we now establish some consequences. Where the results in $x$ and $k$ space are essentially equivalent, we usually state only one form.

Consider the trace. Clearly only the index-symmetric parts contribute to this quantity and in fact only the $E$ and $F$ functions are relevant:

$$
S_{\alpha \alpha}(\mathbf{k})=2 E(\mathbf{k})+\left[(\mathbf{e} \cdot \mathbf{k})^{2}-k^{2}\right] F(\mathbf{k}) .
$$

For isotropic turbulence, this reduces further to $2 E(|\mathbf{k}|)$. In $x$ space, Eq. (21) takes the form [31]

$$
R_{\alpha \alpha}(\mathbf{r})=2 \nabla^{2} E(\mathbf{r})+\left[\nabla^{2}-(\mathbf{e} \cdot \nabla)^{2}\right] \nabla^{2} F(\mathbf{r}) .
$$

Now, for each of the "primary" tensors, its trace is either a pseudoscalar or a scalar. For example, $R_{\alpha \alpha}^{v}(\mathbf{r})=\left\langle\mathbf{v} \cdot \mathbf{v}^{\prime}\right\rangle$ is clearly a true scalar, whereas $R_{\alpha \alpha}^{ \pm}(\mathbf{r})=\left\langle\mathbf{v} \cdot \mathbf{b}^{\prime} \pm \mathbf{b} \cdot \mathbf{v}^{\prime}\right\rangle$ are both pseudoscalars. It follows, by the quotient rule for tensors [32], that $E_{v / b}$ and $F_{v / b}$ must be true scalars and $E^{ \pm}$and $F^{ \pm}$pseudoscalars, in accord with theorem AA. Note that the true or pseudoscalar character of the generating functions is unrelated to their even or oddness under $\mathbf{r} \mapsto-\mathbf{r}$ (see below).

Writing the scalar functions in terms of the poloidal and toroidal functions is also revealing. Referring back to the $k$-space derivation of the $S^{ \pm}$tensors, we have

$$
E^{ \pm}=\left[\left\langle T_{v}^{*} T_{b}\right\rangle \pm \text { c.c. }\right]\left[1-(\mathbf{e} \cdot \hat{\mathbf{k}})^{2}\right] k^{2},
$$

$$
\begin{gathered}
F^{ \pm}=\left\langle T_{v}^{*} T_{b}-P_{v}^{*} P_{b}\right\rangle \pm \text { c.c. }, \\
2 C^{\mp}=\left\langle P_{v}^{*} T_{b}-T_{v}^{*} P_{b}\right\rangle \mp \text { c.c. }, \\
2 H^{ \pm}=\left[\left\langle P_{v}^{*} T_{b}+T_{v}^{*} P_{b}\right\rangle \pm \text { c.c. }\right]\left[1-(\mathbf{e} \cdot \hat{\mathbf{k}})^{2}\right] k,
\end{gathered}
$$

where c.c. denotes the complex conjugate. It follows immediately that the four plus functions are all purely real scalar functions, while the minus forms are pure imaginary. Referring to Eq. (20), it can now be seen that the symmetric parts of $S^{+}\left(S^{-}\right)$are all purely real (imaginary), with the reverse applying for the antisymmetric pieces. This result is obtained independently in Appendix A (see Table II).

By letting the $b$ subscript on the poloidal and toroidal functions become a $v$ and ignoring the c.c. terms, we recover the scalar generating functions for $S^{v}$. In this case $E_{v}, F_{v}$, and $H_{v}$ are pure real, whereas $C_{v}$ is pure imaginary. Identical results hold for $S^{b}$. As expected, these results are also in agreement with those summarized in Table II. Moreover, this analysis reveals that $E_{v}$ is essentially the autocorrelation of the toroidal potential $\left\langle T_{v}^{*} T_{v}\right\rangle$ and $F_{v}$ the difference between the toroidal and poloidal autocorrelations. Similarly, the real and imaginary parts of the poloidal-toroidal cross correlation $\left\langle P_{v}^{*} T_{v}\right\rangle$ are, respectively, $H_{v}$ and $C_{v}$.

As noted at the start of this section, the true vector nature of $\mathbf{v}$ requires that its poloidal and toroidal scalar functions are, respectively, true and pseudoscalars. For $\mathbf{b}$ the situation is obviously reversed. It is then straightforward to show that $E^{ \pm}$and $F^{ \pm}$are pseudoscalars, while $C^{\mp}$ and $H^{ \pm}$are true scalars, with the reverse holding for the $v / b$ forms. Table III summarizes many properties of the generating scalar functions, along with some related ones for the multiplying parts with indices. The results are clearly in accord with theorem AA and this explicit construct represents, in effect, an alternative proof.

\section{PHYSICAL INTERPRETATIONS OF THE SCALAR GENERATING FUNCTIONS}

In this section $\mathbf{v}$ and $\mathbf{b}$ represent the fluctuating portions of the velocity and magnetic field (in Alfvén speed units) of a homogeneous turbulent incompressible magnetofluid. The physical content of the various tensors is then of interest and here we examine such information.

Consider first the $v$ and $b$ tensors. The antisymmetric components are of the form

$$
J_{i j}^{v / b}(\mathbf{k})=i \epsilon_{i j \alpha} k_{\alpha} H_{v / b}(\mathbf{k}),
$$

so that, as is well known $[8,15,25], H$ corresponds to twice the helicity spectrum of the defining field. Integrating over all $\mathbf{k}$ we obtain, for example,

$$
H_{v}(\mathbf{r}=0)=\langle\boldsymbol{\psi} \cdot \mathbf{v}\rangle=\int H_{v}(\mathbf{k}) d^{3} \mathbf{k},
$$

which is twice the bulk helicity of the velocity field. A similar result holds for the magnetic field fluctuations. Note that because $H_{v / b}$ is an even function of $\mathbf{k}$, the integral of $J_{i j}^{v / b}(\mathbf{k})$ over all $\mathbf{k}$ is identically zero. In $x$ space this result takes the form $J_{i j}^{v / b}(\mathbf{r}=0)=0$. Recalling the definitions, it is 
TABLE III. Summary of the properties of the generating scalar functions and their multiplying "parts with indices," for each of the "primary" tensors. " $R$ "' and " $I$ " indicate the real or imaginary nature of the term, " $E$ " and " $\mathrm{O}$ " its even or odd nature under $\mathbf{k} \mapsto-\mathbf{k}$, and " $\mathrm{T}$ " and " $\mathrm{P}$ " its true or pseudotensor character. The final column is the net result for the entire term. See Eq. (20), for example, and the text.

\begin{tabular}{lcccc}
\hline \hline Term & $\begin{array}{c}\text { Scalar } \\
\text { function }\end{array}$ & $\begin{array}{c}\text { Part with } \\
\text { indices }\end{array}$ & $\begin{array}{c}\text { Index } \\
\text { symmetry }\end{array}$ & $\begin{array}{c}\text { Net } \\
\text { character }\end{array}$ \\
\hline$E_{v / b}$ & RET & RET & symmetric & RET \\
$F_{v / b}$ & RET & RET & symmetric & RET \\
$C_{v / b}$ & IOP & IOP & symmetric & RET \\
$H_{v / b}$ & REP & IOP & antisymmetric & IOT \\
$E^{+}\left(E^{-}\right)$ & REP (IOP) & RET & symmetric & REP (IOP) \\
$F^{+}\left(F^{-}\right)$ & REP (IOP) & RET & symmetric & REP (IOP) \\
$C^{-}\left(C^{+}\right)$ & IOT (RET) & IOP & symmetric & REP (IOP) \\
$H^{+}\left(H^{-}\right)$ & RET (IOT) & IOP & antisymmetric & IOP (REP) \\
\hline \hline
\end{tabular}

clear that this must be the case since the $R_{i j}^{v / b}$ are manifestly symmetric at $\mathbf{r}=\mathbf{0}$. Physically, the magnetic helicity consists of two distinct contributions: (i) the topological linkage and (ii) the twist of magnetic field lines (or flux tubes) $[12,26,27]$. Similar statements can be made for the velocity helicity $H_{v}$.

What about the three scalar functions associated with the symmetric parts of $S^{v / b}(\mathbf{k})$ ? Suppose the turbulence is isotropic, so that

$$
S_{i j}^{v / b}(\mathbf{k})=\left(\delta_{i j}-\frac{k_{i} k_{j}}{k^{2}}\right) E_{v / b}(|\mathbf{k}|)+i \epsilon_{i j \alpha} k_{\alpha} H_{v / b}(|\mathbf{k}|)
$$

Thus $E_{v / b}(|\mathbf{k}|)$ is equal to $4 \pi k^{2}$ times the omnidirectional energy spectrum (see, e.g., [4]) and the scalar functions $F_{v / b}$ and $C_{v / b}$ are associated with departures from isotropy. An examination of the trace (21) indicates that for nonisotropic turbulence $F_{v / b}$ contributes to the energy spectrum, but $C_{v / b}$ does not.

The physical correlations implied by $C_{v}$ and $C_{b}$ are clearly less familiar than those associated with energy or helicity. However, just as we were able to show (in Sec. III B) that the generating function associated with an antisymmetric autocorrelation tensor is associated with specific nonvanishing one-point pseudoscalar correlations, here we can provide examples of correlations associated with nonvanishing $C_{v}$ or $C_{b}$. For example,

$$
\begin{aligned}
2\left[\nabla^{2}-(\mathbf{e} \cdot \nabla)^{2}\right]^{2} C_{v / b}(\mathbf{r}) & =e_{i} e_{l} \boldsymbol{\epsilon}_{l \alpha j} \partial_{\alpha}\left[R_{i j}(\mathbf{r})+R_{j i}(\mathbf{r})\right] \\
& =\langle\mathbf{e} \cdot \mathbf{v}(\mathbf{x})[\boldsymbol{\omega}(\mathbf{x}+\mathbf{r})-\boldsymbol{\omega}(\mathbf{x}-\mathbf{r})] \cdot \mathbf{e}\rangle .
\end{aligned}
$$

Clearly this correlation is a pseudoscalar and when nonzero implies a nonvanishing $C_{v / b}(\mathbf{r})$. In addition, we know [cf. Sec. V C, Eq. (25), and Table III] that $C$ is associated with a nonzero imaginary part of the cross correlation between the toroidal and poloidal potentials.

It is useful to elaborate upon the discussion at the end of Sec. IV to reveal the physical nature of the $C$-type correlations. Consider a fixed wave vector $\mathbf{k}$ and its associated Fourier amplitude for one of the turbulent fields, say $\mathbf{b}$. Let us return, for the moment, to a Cartesian coordinate system in which the $\hat{\mathbf{z}}$ direction is aligned with $\mathbf{k}$. There are many such coordinate systems, distinguished by rotations about the $\mathbf{k}$ axis. If the plane is spanned by axes aligned with $\mathbf{e} \times \mathbf{k}$ and $\mathbf{k} \times(\mathbf{e} \times \mathbf{k})$, for arbitrary reference direction $\mathbf{e}$, then the change from one $\mathbf{k}$-aligned system to another is accomplished by selection of a particular e. As explained in Sec. IV, in this frame contributions to the correlation tensor by excitations at wave vector $\mathbf{k}$ take the simple form expressed in Eq. (11). Contributions to the real-space quantities by excitations at $\mathbf{k}$ can be written as (the real part of) $\mathbf{b}_{\mathbf{k}}(\mathbf{x})=\left(B_{x}, B_{y}\right) e^{i k z}$, where the two component complexvalued vector $\left(B_{x}, B_{y}\right)$ lies in the plane perpendicular to $\mathbf{k}$. This is a familiar one-dimensional "slab" geometry; every Fourier contribution looks like a slab fluctuation in its special coordinate frame. Considering the hodographic projection of $\mathbf{b}_{\mathbf{k}}(\mathbf{x})$ onto this plane, we see that in general the tip of the field vector traces an ellipse. There will be a preferred coordinate system in the $x-y$ plane that selects the principle axes of this ellipse. For the right choice of e, there are no indexsymmetric off-diagonal terms needed to describe the correlation. Everything about the correlations produced in this way is specified by the major and minor radii of the ellipse (the energies in the associated $x$ and $y$ directions) and the phase lag between these components (i.e., the magnetic helicity). Thus, for this $\mathbf{k}$, contributions to $C$ cannot appear because all the information is accounted for, using three numbers. However, unless all wave vectors have polarizations that align in a single Cartesian frame (a highly unlikely circumstance), we cannot eliminate all contributions to $C$ in this way. A physical interpretation of $C$, therefore, is that it represents information about the orientation of elliptical polarizations for the various wave vectors. In isotropic turbulence, for every $\mathbf{k}$ the ellipses become circles (regardless of the helicity) and every choice of $\mathbf{e}$ diagonalizes the symmetric part of the correlations, so $C=0$.

It is interesting to note that the helicity can also be eliminated locally in $\mathbf{k}$ space in a similar way by choosing a complex (polarization) coordinate system to describe the $x-y$ plane perpendicular to $\mathbf{k}$. In this special frame the phase lag between $B_{x}$ and $B_{y}$ in the local slab system is absorbed and only two independent numbers remain (right and left circularly polarized energies), apart from an unimportant overall phase. Once again, however, the polarization basis 
set will usually be different for various wave vectors. In general, then, four independent scalars are required.

Consider next the plus tensor. From the definitions of $R_{i j}^{+}$and the cross helicity $H_{c}$, it follows that [33]

$$
2 H_{c}=R_{\alpha \alpha}^{+}(\mathbf{r}=0)=\int I_{\alpha \alpha}^{+}(\mathbf{k}) d^{3} \mathbf{k},
$$

so that $I_{\alpha \alpha}^{+}(\mathbf{k})=2 E^{+}(\mathbf{k})+\left[(\mathbf{e} \cdot \mathbf{k})^{2}-k^{2}\right] F^{+}(\mathbf{k})$ is twice the cross helicity spectrum. The cross helicity is also interpretable as a linkage [26]. Specifically, it is a measure of that between vorticity tubes and magnetic flux tubes. Another interpretation of the cross helicity is that it is proportional to the (average) correlation between $\mathbf{v}$ and $\mathbf{b}$. Hence it is convenient to introduce the normalized cross helicity $\sigma_{c}=2 H_{c} / E^{\text {tot }}$, which is bounded by \pm 1 and also expressible in terms of elements of the primary tensors. The extremal values correspond to $\mathbf{v}= \pm \mathbf{b}$ and are associated with exact cancellation of the nonlinear terms in the (incompressible) MHD equations of motion. This type of Alfvénic fluctuation is frequently observed in the solar wind plasma (see, e.g., [24]).

By direct analogy with the $v$ and $b$ tensors, we refer to $H^{+}(\mathbf{k})$ as (twice) the spectrum of the "helicity of the cross helicity" since it has the same mathematical relation to $H_{c}(\mathbf{k})$ as does $H_{b}(\mathbf{k})$ to $E^{m}(\mathbf{k})$ [20]. However, this helicity of the cross helicity is somewhat different in nature than the velocity and magnetic helicities. It is still an even function of $\mathbf{k}$, as is $H_{b} \equiv 2 H_{m}$, for example, so that in general there is a bulk value as well as a spectrum, but it is a true scalar rather than a pseudoscalar.

Finally, we turn to the physical content of the minus tensor. From the definition (4) it follows that

$$
R_{i j}^{-}(\mathbf{r}=0)=\left\langle v_{i} b_{j}-b_{i} v_{j}\right\rangle,
$$

which is related to the ensemble-averaged induced electric field (emf) of the fluctuations $\mathcal{E}=-\langle\mathbf{v} \times \mathbf{b}\rangle=-\nabla \Phi$. The function $\Phi(\mathbf{r}) \equiv H^{-}(\mathbf{r})$ is the electric potential. In fact, it is not hard to show that the electric field is contained only in the antisymmetric components

$$
J_{i j}^{-}(\mathbf{r}=0)=-\epsilon_{i j \alpha} \mathcal{E}_{\alpha}=\left.\epsilon_{i j \alpha} \frac{\partial \Phi}{\partial r_{\alpha}}\right|_{\mathbf{r}=0} .
$$

Consequently, in $k$ space $\Phi(\mathbf{k}) \equiv H^{-}(\mathbf{k})$ is interpretable as the spectrum of the electric potential. As will be shown in Sec. VIII, this result enables information on the spectral anisotropy of the electric field to be obtained from the reduced spectra of $J_{i j}^{-}(\mathbf{k})$.

When the turbulence is isotropic, but not necessarily mirror symmetric (see also Sec. VII), the induced electric field vanishes. Mathematically this follows since $H^{-}(\mathbf{k})$ must then be a function of $k^{2}$, but is also explicitly odd and the only function that satisfies both these conditions is $H^{-}(\mathbf{k})=0$. This behavior is opposite to that of the helicities of the $v / b$ tensors $H_{v / b}$, which must vanish for completely mirror-symmetric turbulence, but do not necessarily vanish for isotropic geometries. They are even pseudoscalar functions, whereas $\mathrm{H}^{-}$is an odd true scalar function. It follows that the presence of an induced (ensemble-averaged) electric field is associated with the anisotropy of the turbulence. This result is likely to be of interest in mean-field dynamo theory (see, e.g., [11]), particularly in connection with the $\alpha$-dynamo closure.

Although $\langle\mathbf{v} \times \mathbf{b}\rangle$ vanishes for isotropic turbulence, the mean value of the square $\mathcal{E}_{r m s}^{2}=\left\langle(\mathbf{v} \times \mathbf{b})^{2}\right\rangle$ is in general nonzero. We can evaluate $\mathcal{E}_{r m s}^{2}$ under these conditions, with the additional assumptions that (i) all components $v_{i}$ and $b_{i}$ are Gaussian random variables and (ii) unless $i=j$, then $\left\langle v_{i} b_{j}\right\rangle=0,\left\langle v_{i} v_{j}\right\rangle=0$, and $\left\langle b_{i} b_{j}\right\rangle=0$. It can then be shown that

$$
\mathcal{E}_{r m s}^{2}=\frac{2}{3}\left[\left\langle v^{2}\right\rangle\left\langle b^{2}\right\rangle-\langle\mathbf{v} \cdot \mathbf{b}\rangle^{2}\right]
$$

The rms value is thus prescribed, not just bounded, once the energies and the cross helicity are given. Clearly, $\mathcal{E}_{r m s}$ is maximal when the cross helicity is zero. Note, however, that in fully developed homogeneous turbulence the components of $\mathbf{v}$ and $\mathbf{b}$ are unlikely to be distributed as Gaussian random variables.

Taking into account the anomalous nature of the minus tensor, Zhou and Matthaeus [20] have suggested that its symmetric parts contain the "helicity of the electric field." In particular they refer to the trace of the minus tensor

$$
S_{\alpha \alpha}^{-}(\mathbf{k})=2 i \operatorname{Im}\left\langle\mathbf{v}^{*} \cdot \mathbf{b}\right\rangle=2 E^{-}(\mathbf{k})-\left[(\mathbf{e} \cdot \mathbf{k})^{2}-k^{2}\right] F^{-}(\mathbf{k})
$$

by this name. The integral of this quantity over all wave vectors is identically zero. In $x$ space this is the obvious statement $R_{\alpha \alpha}^{-}(0)=\langle\mathbf{v} \cdot \mathbf{b}-\mathbf{b} \cdot \mathbf{v}\rangle=0$, so that despite having a nonzero spectrum, the helicity of the electric field always has a bulk value of zero. As shown in Sec. VII, for isotropic turbulence the traced spectrum (35) is also zero, a property that may be tested for when analyzing observational or experimental data.

To summarize, for incompressible homogeneous turbulence, with arbitrary rotational symmetry, each of the following quantities, or equivalently its spectrum, is generated by a single scalar function: velocity helicity, magnetic helicity, helicity of the cross helicity, and the induced electric field. In each case, the quantity is associated with the antisymmetric portion of its correlation function or spectral tensor. Recall also that the magnetic helicity is a rugged invariant of ideal magnetohydrodynamics. The symmetric pieces of the $v / b$ spectral tensors hold the associated energy spectra, their (traced) sum forming the spectra of another rugged invariant (total energy). The third rugged invariant, cross helicity, is contained in the trace of the + tensor. Unfortunately, the quantities associated with the symmetric parts of the - tensor, while undoubtedly physical in character, are currently less well understood.

\section{SPECIAL CASES}

We now present some specializations of the above results for particular symmetries of the turbulence. It will be most convenient to do so using the $k$-space forms of Sec. V and in particular the tensor form given by Eq. (20). We will drop the \pm labels, however, noting that the results hold for all 
four of the "primary" spectral tensors, unless we explicitly indicate otherwise. Be aware that the parity of the scalar functions may differ from tensor to tensor (see Table III). We begin each subsection with a precise definition of the symmetry.

\section{A. Isotropy}

Turbulence is isotropic if all averaged quantities depending on $\mathbf{v}$ and $\mathbf{b}$ are invariant under arbitrary (proper) rotations of the $\mathbf{v}$ and $\mathbf{b}$ fields about arbitrary axes. Consequently, there can be no dependence on any preferred direction(s). All terms in Eq. (20) involving e must therefore vanish, that is, $F=C=0$, leading to the well-known result, which may be read off with ease from Eq. (20), e.g., [4]. Isotropy also requires that the scalar functions depend only on the wavevector magnitude $k=|\mathbf{k}|$. However, the minus scalar functions and $C_{v / b}$ are explicitly odd in $\mathbf{k}$ and hence must also be identically zero in this case. It follows that the full - spectral tensor and its real space counterpart $R_{i j}^{-}(\mathbf{r})=\frac{1}{2}\left\langle v_{i} b_{j}^{\prime}-b_{i} v_{j}^{\prime}\right\rangle$ are identically zero for isotropic turbulence since each of its scalar functions vanishes. Thus only six independent scalar functions are needed to specify isotropic turbulence: an $E$ and an $H$ for each of the $v / b$ and + tensors. This is to be contrasted with the general case where 16 such functions are required.

In all cases the trace reduces to dependence on a single scalar function: $S_{\alpha \alpha}(k)=2 E(k)$. The trace of the minus tensor is identically zero, so that there is no helicity of the electric field in isotropic turbulence. Note that, except for the minus tensor, isotropic turbulence does not preclude the presence of helicity $H$. As has been remarked upon, the vanishing of $\mathrm{H}^{-}$for isotropic turbulence means that no ensemble-averaged induced electric field exists in the absence of preferred directions.

\section{B. Mirror symmetry}

Turbulence is mirror, or reflection, symmetric with respect to a point (or a plane) if all averaged quantities depending on $\mathbf{v}$ and $\mathbf{b}$ are invariant under reflection of these fields through the point (or the plane). Thus the correlation tensors associated with mirror-symmetric turbulence can contain, only terms exhibiting overall even parity in $\mathbf{k}$ [note that while earlier workers (see, e.g., $[2,4,7,9]$ ) included mirror symmetry in the definition of isotropy, this is not current practice]. Thus the three helicities $H_{v}, H_{b}$, and $H^{+}$that are a measure of mirror asymmetry are all identically zero, as are $E^{-}, F^{-}$, and $C^{+}$(Table III).

As far as the trace is concerned, the "normal" tensors are unaffected by the presence or absence of mirror symmetry. In stark contrast to this, $S_{\alpha \alpha}^{-}$collapses to zero when mirror symmetry is imposed. Indeed, for the minus tensor, the only term that remains is the antisymmetric piece, which has been shown to contain the spectrum of the electric potential. The anomalous nature of the minus tensor is particularly clearly revealed when we consider turbulence that is mirror symmetric.

\section{Axisymmetry and the presence of a mean field}

Turbulence is axisymmetric (with respect to a fixed axis) if all averaged quantities depending on $\mathbf{v}$ and $\mathbf{b}$ are invariant under arbitrary rotations of these fields about the fixed axis. The general results of Sec. V require only slight modifications for such flows. It is convenient to restrict $\mathbf{e}$ to be the axis of symmetry, with the scalar generating functions then depending only upon the variables $(r, \mathbf{r} \cdot \mathbf{e})$ or equivalently $\left[(\mathbf{r} \times \mathbf{e})^{2}, \mathbf{r} \cdot \mathbf{e}\right]$. The parts with indices are independent of the true or pseudovector nature of $\mathbf{e}$ (components of $\mathbf{e}$ always appear in product pairs), so the overall structure is the same whether the preferred direction is associated with a mean flow, a uniform magnetic field, or a rotation axis, for example. However, the transformation properties of $\mathbf{e}$ do place restrictions on the functions appearing in the tensors in order that the required parity under inversion be maintained. Bearing these conditions in mind, the present results easily reduce, in the special case of axisymmetry, to the form presented by Matthaeus and Smith [8]. The axisymmetric correlation tensor presented by Moffatt [12] is formally correct, but includes too many independent scalars. Presumably this is due to an incomplete use of the solenoidal constraint. In particular the antisymmetric autocorrelation is described as containing three scalar functions, whereas only one is required [8,15] (Moffatt and Proctor [25] subsequently state without proof the result that only one such function is required in general).

Chandrasekhar [7] showed that axisymmetric systems that are also mirror symmetric have only two independent scalar generating functions (for normal correlation tensors). In our notation this corresponds to the vanishing of the $C$ and $H$ functions.

\section{Two-dimensional symmetry}

Turbulence has two-dimensional (2D) symmetry (with respect to a single fixed direction) if all averaged quantities depending on $\mathbf{v}$ and $\mathbf{b}$ are independent of coordinates parallel to the fixed direction. In $k$ space this constrains all excited wave vectors to be perpendicular to the axis of symmetry (again taken to be parallel to e), so that $S_{i j}(\mathbf{k})$ is zero unless $\mathbf{e} \cdot \mathbf{k}=0$. In the case of the reduced MHD description the collapse to near two dimensionality is associated with a preferred direction induced by a strong uniform magnetic field $[34,35]$. For the two-dimensional case, Eq. (20) then reduces to

$$
\begin{aligned}
S_{i j}(\mathbf{k})= & {\left[\delta_{i j}-\frac{k_{i} k_{j}}{k^{2}}\right] E(\mathbf{k})-e_{i} e_{j} k^{2} F(\mathbf{k})-i\left[e_{i}(\mathbf{e} \times \mathbf{k})_{j}\right.} \\
& \left.+e_{j}(\mathbf{e} \times \mathbf{k})_{i}\right] k^{2} C(\mathbf{k})+i \epsilon_{i j \alpha} k_{\alpha} H(\mathbf{k}),
\end{aligned}
$$

where $\mathbf{k} \equiv \mathbf{k}_{\perp}$, the wave vector in the plane perpendicular to e. (This restriction can also be accomplished using Dirac $\delta$ functions.) Note that we may have helicity, but it influences only those correlations that involve exactly one field component in the e direction. The axisymmetric two-dimensional model is now obtained by further specializing to scalar functions that depend only upon the magnitude of $\mathbf{k}_{\perp}$.

The above model is sometimes called " $2 \frac{1}{2} \mathrm{D}$ " because it involves two components of wave vector but three components of the field. Insisting that the fluctuation amplitudes are also perpendicular to e yields the spectral tensor appropriate for the usual definition of 2D turbulence, wherein all activity 
is confined to a plane with normal vector $\mathbf{e}$,

$$
S_{i j}(\mathbf{k})=\left[\delta_{i j}-\frac{k_{i} k_{j}}{k^{2}}-e_{i} e_{j}\right] E(\mathbf{k}) .
$$

This is simplest to see by considering a particular Cartesian coordinate system, with $\mathbf{e}$ in the 3 direction, say. Then, since $k_{3}=0$, it follows that $S_{33}=E-k^{2} F$, and as this is energy in components parallel to the symmetry axis we require it to be zero. Consideration of $S_{23}$ and $S_{32}$ leads to the conclusion that $H=C=0$. Physically, this geometry is believed to be relevant in situations where a strong uniform magnetic field threads a turbulent (magneto)fluid [34-39]. Even for the nonaxisymmetric case, however, the autocorrelation tensor for (say) the magnetic field fluctuation, in this pure 2D turbulence, is completely specified by a single scalar function.

\section{E. Slab symmetry}

Finally, we consider the slab geometry. This is a particular case of axisymmetric turbulence, where the excited wave vectors are parallel to $\mathbf{e}$, so that $\mathbf{e} \cdot \mathbf{k}= \pm k$ and $\mathbf{e} \times \mathbf{k}=0$. For such symmetries, all dependence on $C$ drops out and, considering again the coordinate system with $\mathbf{e}$ in the 3 direction, it can be shown that the factor multiplying $F$ is always zero. Hence only the energy and helicity spectra contribute to the slab spectral tensor(s):

$$
S_{i j}(\mathbf{k})=\left[\delta_{i j}-\frac{k_{i} k_{j}}{k^{2}}\right] E(\mathbf{k})+i \epsilon_{i j \alpha} k_{\alpha} H(\mathbf{k}) .
$$

In magnetohydrodynamics, slab fluctuations often correspond to (large-amplitude) Alfvén waves propagating along a uniform magnetic field parallel to e. Such waves are exact solutions of the incompressible nondissipative MHD equations and have been observed in laboratory and space plasmas (see, e.g., [24]). Recent evidence has indicated that MHD turbulence in the solar wind may be reasonably well described as a superposition of slab and 2D fluctuations [18]. Differences in the structural form of the 2D and slab correlation tensors permit direct observational evaluation of this hypothesis [40].

\section{MEASUREMENT ISSUES}

Some results of the previous sections are now considered within the context of data analysis. Our main results are expected to be of very broad applicability since we have presented what we believe to be the most general form of the second-rank homogeneous MHD correlations and as such we do not expect to anticipate all eventual applications at this time. However, we presently have in mind specific applications involving properties of the solar wind, for which extensive plasma and magnetic field datasets are available.

A general result, pertinent to all four basic tensors, is that the index-antisymmetric part of each spectral tensor $\left(S^{v / b}, S^{ \pm}\right)$is of the form $i \epsilon_{i j \alpha} k_{\alpha} H(\mathbf{k})$, which involves only one scalar. The function $H$ is usually a proper scalar (except for $S^{-}$when it is pseudoscalar) and in all cases its reduced spectrum (and bulk value) are obtained easily from singlepoint, frozen-in measurements, which provide the values of the tensor correlations for separation vectors along a single Cartesian direction, $\hat{\mathbf{x}}_{1}$, say. The method for measurement is the simple generalization of the technique for extraction of reduced magnetic helicity spectra [15] and is summarized by the formula

$$
H^{r e d}\left(k_{1}\right)=\frac{\operatorname{Im}\left\{S_{23}\left(k_{1}\right)\right\}}{k_{1}},
$$

where $H^{r e d} \equiv \int H(\mathbf{k}) d k_{2} d k_{3}$. As with the original helicity formula, this is valid for homogeneous turbulence with arbitrary rotational symmetry.

A quantity of the above type that is of particular interest is the induced electric field. As shown in Sec. VI, the (mean) electric field induced by the fluctuating velocity and magnetic fields is related to the antisymmetric part of the minus tensor, i.e., $J_{i j}^{-}(\mathbf{r}=\mathbf{0})=-\epsilon_{i j \alpha} \mathcal{E}_{\alpha}$, with $\mathcal{E}_{\alpha}=-\nabla_{\alpha} \Phi(\mathbf{r})$. In terms of the spectral tensor, $\mathcal{E}_{\alpha}(\mathbf{k})=-i k_{\alpha} \Phi(\mathbf{k})$, with $H^{-}(\mathbf{k}) \equiv \Phi(\mathbf{k})$ the spectrum of the electric potential. Hence knowledge of the off-diagonal components of $R_{i j}^{-}$yields the induced electric field. Values at separation $\mathbf{r}=\mathbf{0}$ are the components of the average induced electric field, while the correlations at nonzero $\mathbf{r}$ can be thought of as a correlation function associated with the electric field. [Note that the terminology "electric field correlation function" would ordinarily be associated with a fourth-rank correlation (see below).] Given data intervals of sufficient length and quality, it is straightforward to calculate the appropriate correlation tensor and then extract the induced emf (see, e.g., [41]). However, it seems not to have been properly appreciated that the separate components of the induced electric field are related to the single underlying fundamental quantity $\Phi(\mathbf{k})$. In fact, because of this structure the induced electric field provides additional information in homogeneous turbulence. As we now show, the spectrum of $J^{-}$can be used to construct a measure of the spectral anisotropy of $\mathcal{E}$.

From Eq. (20) we have $J_{23}^{-}(\mathbf{k})=i k_{1} H^{-}(\mathbf{k})$, etc. Integrating this last equation over all $\mathbf{k}$ yields $J_{23}^{-}(\mathbf{r}=\mathbf{0})=-\mathcal{E}_{1}$. Now suppose that the observation direction and the 1 direction coincide. Integrating (reducing) over the 2 and 3 directions then gives

$$
\Phi^{r e d}\left(k_{1}\right)=-\frac{i}{k_{1}} \int J_{23}^{-}(\mathbf{k}) d k_{2} d k_{3}=-i \frac{J_{23}^{-, r e d}}{k_{1}} .
$$

Moreover, reducing the $J_{12}^{-}$and $J_{31}^{-}$components yields quantities that can be interpreted as mean wave numbers associated with the directional components of the electric field. For example, a mean $k_{3}$ can be defined as

$$
\bar{k}_{3}=\frac{\int k_{3} \Phi(\mathbf{k}) d k_{2} d k_{3}}{\int \Phi(\mathbf{k}) d k_{2} d k_{3}}=k_{1} \frac{J_{12}^{-, r e d}\left(k_{1}\right)}{J_{23}^{-, r e d}\left(k_{1}\right)} .
$$

Similarly, we can define $\bar{k}_{2} / k_{1}=J_{31}^{-, \text {red }}\left(k_{1}\right) / J_{23}^{-, \text {red }}\left(k_{1}\right)$. Thus, because the underlying fields are solenoidal, it is possible to obtain information about the spectrum of the electric field in directions perpendicular to the measurement direction. For practical situations it is not yet clear how best to 
normalize these quantities. We are attempting to extract such information from some available solar wind datasets.

It was also shown in Sec. VI that for isotropic turbulence the induced electric field vanishes. In addition, the induced electric field spectrum, as defined above, also vanishes exactly for isotropic fluctuations. The simplest way to see this is to notice that the electric potential spectrum $\Phi(\mathbf{k})$ is odd in $\mathbf{k}$. For isotropic fluctuations, this scalar must be a function of $|\mathbf{k}|$ only. Thus it vanishes. The same conclusion is obtained in ordinary space, where one invokes the property that $\Phi(\mathbf{r})$ is odd in $\mathbf{r}$, while isotropy demands that the same function depend only upon $|\mathbf{r}|$. In addition, if the fluctuations are Gaussian, then $\mathcal{E}_{r m s}$ is not an independent parameter. Comparing these theoretical predictions for $\mathcal{E}$ and $\mathcal{E}_{\text {rms }}$ to observationally determined values may provide a useful measure of the extent to which the fluctuations depart from isotropic and Gaussian distributions.

In an analysis of solar wind fluctuations in terms of Elsässer variables Tu et al. [42] found that frequency spectra of the helicity of the electric field, which they denote by $e^{S}(f)$, equivalent to the reduced form of our $S_{\alpha \alpha}^{-}(\mathbf{k})$, have both positive and negative contributions and that there is also a fairly wide scatter in the data points, no clean power laws being evident. The presence of both signs in such spectra is of course necessary to ensure that when the integration over all wave numbers (frequencies) is performed, the net result will be zero, in accordance with the results of Sec. VI.

\section{DISCUSSION}

In this paper we have presented the complete structure of second-rank Cartesian correlation tensors involving a solenoidal vector field $\mathbf{v}$ and a solenoidal pseudovector field $\mathbf{b}$. Four basic correlation tensors need to be described, involving the autocorrelations and the properly symmetrized and antisymmetrized cross correlations. Each of the basic correlation tensors depends upon exactly four underlying scalar (or pseudoscalar) functions, sometimes known as generating functions. In each case precisely one generating function is connected with the index-antisymmetric part of the correlation tensor and three are associated with the symmetric portion.

In the course of this development, we have examined carefully several important misleading or incorrect statements that have remained uncorrected in the literature of the theory of correlation tensors for homogeneous (or isotropic) turbulence. Most of these problems have arisen because of confusion over the circumstances in which the generating scalar functions can be, or must be, pseudoscalars. We also described how pseudoscalar functions arise from one-point correlations, such as helicities.

Although these results are of a general nature, our intention is to associate the fields $\mathbf{b}$ and $\mathbf{v}$ with the magnetic and (incompressible) velocity fields, respectively, that appear in magnetohydrodynamics turbulence. The general correlations presented here provide compact and complete Cartesian representations of the spectra and two-point correlation functions of relevance in MHD. A number of bulk quantities of interest are also described by the tensors.

For axisymmetric homogeneous turbulence we have shown that the true or pseudotensor character of the pre-
TABLE IV. Some correlation functions and spectral matrices in terms of Elsässer variables. Note that these matrices typically are neither a pure tensor nor a pure pseudotensor. $R^{D}=R^{v}-R^{b}$ is the energy difference tensor [20,49-51].

\begin{tabular}{lccc}
\hline \hline Symbol & Definition & $v-b$ form & $\begin{array}{c}\text { Homogeneity } \\
\text { condition }\end{array}$ \\
\hline$H_{i j}^{ \pm}(\mathbf{r})$ & $\left\langle z_{i}^{ \pm} z_{j}^{ \pm \prime}\right\rangle$ & $R_{i j} \pm 2 R_{i j}^{+}$ & $H_{i j}^{ \pm}(\mathbf{r})=H_{j i}^{ \pm}(-\mathbf{r})$ \\
$\Lambda_{i j}(\mathbf{r})$ & $\left\langle z_{i}^{+} z_{j}^{-\prime}\right\rangle$ & $R_{i j}^{D}-2 R_{i j}^{v b,-}$ & $\Lambda_{i j}(\mathbf{r})=\widetilde{\Lambda}_{i j}(-\mathbf{r})$ \\
$\widetilde{\Lambda_{i j}}(\mathbf{r})$ & $\left\langle z_{i}^{+\prime} z_{j}^{-}\right\rangle$ & $R_{j i}^{D}+2 R_{j i}^{v b,-}$ & \\
$F_{i j}(\mathbf{r})$ & $\left\langle\Lambda_{i j}+\widetilde{\Lambda_{i j}}\right\rangle$ & $2 I_{i j}^{D}-4 J_{i j}^{-}$ & $F_{i j}(\mathbf{r})=F_{i j}(-\mathbf{r})$ \\
$G_{i j}(\mathbf{r})$ & $\left\langle\Lambda_{i j}-\widetilde{\Lambda_{i j}}\right\rangle$ & $2 J_{i j}^{D}-4 I_{i j}^{-}$ & $G_{i j}(\mathbf{r})=-G_{i j}(-\mathbf{r})$ \\
\hline \hline
\end{tabular}

ferred direction is irrelevant as far as the structure of the correlation tensors is concerned. This follows because components of e only appear in product pairs and as such are immune to an overall sign change of $\mathbf{e}$. This result, as well as the statement we call theorem AA, corrects earlier work [8] and restores the intuitive idea of dependence on a single direction being a unique geometrical concept.

Most of the above results may also be expressed in terms of Elsässer variables. Clearly, this may be accomplished by either (a) substituting $\mathbf{z}^{ \pm}=\mathbf{v} \pm \mathbf{b}$ into the final forms given above or (b) starting with correlation functions defined in terms of the $\mathbf{z}^{ \pm}$(e.g., $H_{i j}^{ \pm}=\left\langle z_{i}^{ \pm} z_{j}^{ \pm \prime}\right\rangle$ ) and proceeding from there. These forms may be more convenient in some applications, e.g., for solar wind fluctuations. For completeness, we include in Table IV a summary of some of our main results transcribed to the Elsässer representation. Note that the Elsässer correlation matrices are not proper tensors because they mix contributions that are invariant under the full rotation group with contributions that reverse sign under improper rotations.

\section{ACKNOWLEDGMENTS}

We thank C. W. Smith and J. W. Bieber for enlightening conversations. This work was supported in part by NSF Grant No. ATM-9318728 at Bartol and by the United Kingdom PPARC Grant No. GR/K98711 and the Nuffield Foundation Grant No. SCI/180/94/400.

\section{APPENDIX A: BASIC RESULTS}

For completeness we list here some of the more elementary results, many of which are well known and have been given before, (see, e.g., $[4,8,29]$ ). First, however, it is useful to draw attention to some semantic distinctions. The terms even and odd refer to the behavior of a function under reversal of the spatial separation $\mathbf{r} \rightarrow-\mathbf{r}$. The full operation of coordinate inversion involves not only $\mathbf{r} \rightarrow-\mathbf{r}$, but mapping all vectors that transform like the position vector to their opposites and is equivalent to an improper rotation (reflection followed by rotation). Thus the evenness or oddness ("parity") of a scalar function is distinct from its tensor transformation character. Equivalent definitions hold for the Fourier space forms.

The proper or improper transformation character of the tensors plays an important role in their description. If $\mathrm{M}$ 
represents a change of basis from one Cartesian coordinate system to another, such that $\widetilde{\mathbf{x}}=\mathbf{M} \mathbf{x}$, then a proper tensor field of rank $n, \mathrm{~T}_{j_{1} j_{2} \cdots j_{n}}(\mathbf{x})$, transforms under such a change of basis as

$$
\tilde{\mathrm{T}}_{\alpha_{1} \alpha_{2} \cdots \alpha_{n}}(\widetilde{\mathbf{x}})=\mathrm{M}_{\alpha_{1} j_{1}} \mathrm{M}_{\alpha_{2} j_{2}} \cdots \mathrm{M}_{\alpha_{n} j_{n}} \mathrm{~T}_{j_{1} j_{2} \cdots j_{n}}(\mathrm{Mx})
$$

If this transformation law holds only for a subset of the changes of basis (e.g., rotations but not reflections) then $T$ is an improper (or pseudo) tensor field of rank $n$ (see, e.g., [32]). In this context we will use true and pseudo as synonyms for proper and improper.

Suppose that a correlation function can be written

$$
R_{i j}(\mathbf{r})=\left(\delta_{i j}-\frac{r_{i} r_{j}}{r^{2}}\right) A(\mathbf{r})+\epsilon_{i j \alpha} \frac{\partial}{\partial r_{\alpha}} B(\mathbf{r})+\cdots,
$$

where $\delta_{i j}$ is the Kronecker delta and $\epsilon_{i j \alpha}$ the Levi-Civita permutation tensor. It is convenient to think of each additive term as consisting of an elementary tensor that has indices, multiplied by a (true or pseudo) scalar function of $\mathbf{r}$. Indeed, this can be shown to be the case in general $[1,4]$. The parts with indices are essentially geometrical aspects of the correlation functions. For example, $\delta_{i j}-r_{i} r_{j} / r^{2}$ is an isotropic form in that contraction with two vectors $a_{i}$ and $c_{j}$ produces a true scalar that is invariant under (a) rigid body rotations (of either the coordinate system or the turbulence) and (b) coordinate reflections, provided that the three vectors $\mathbf{r}$, a, and $\mathbf{c}$ all transform like the position vector. However, other isotropic forms can involve pseudoscalars.

Since $\mathbf{v}$ and $\mathbf{b}$ are solenoidal we have

$$
\frac{\partial}{\partial r_{j}} R_{i j}(\mathbf{r})=\frac{\partial}{\partial r_{i}} R_{i j}(\mathbf{r})=0,
$$

or equivalently in $k$ space, $k_{i} S_{i j}(\mathbf{k})=k_{j} S_{i j}(\mathbf{k})=0$. Similar results hold for the $I$ and $J$ tensors, so that the indexsymmetric and -antisymmetric parts are separately solenoidal.

Next, homogeneity requires that

$$
R_{i j}^{v / b}(-\mathbf{r})=R_{j i}^{v / b}(\mathbf{r}), \quad R_{i j}^{ \pm}(-\mathbf{r})= \pm R_{j i}^{ \pm}(\mathbf{r}),
$$

as is easily seen by letting $\mathbf{x} \rightarrow \mathbf{x}-\mathbf{r}$ in the definitions. Note that $R^{-}$is anomalous, containing an overall negative sign. The $I$ 's and $J$ 's each satisfy the same homogeneity condition as their "parent" $R$. The $k$-space forms are obtained by letting $R \rightarrow S$ and $\mathbf{r} \rightarrow \mathbf{k}$.

If $f(\mathbf{x})$ is real then its Fourier transform satisfies the reality condition $f(-\mathbf{k})=f^{*}(\mathbf{k})$, where an asterisk denotes complex conjugation. Using this property and the assumed equivalence of ensemble and space averaging (via invocation of ergodicity), it can be shown that, for example [43],

$$
S_{i j}^{v}(\mathbf{k}) \delta(\mathbf{k}+\mathbf{p})=\left\langle v_{i}(\mathbf{p}) v_{j}(\mathbf{k})\right\rangle .
$$

A consideration of the consequences of homogeneity, index symmetry, and reality for $I_{i j}^{ \pm}(\mathbf{k})$ shows that $I_{i j}^{ \pm *}(\mathbf{k})=I_{i j}^{ \pm}(-\mathbf{k})= \pm I_{i j}^{ \pm}(\mathbf{k})$ and therefore $I^{+}(\mathbf{k})$ is a real index-symmetric even function of $\mathbf{k}$. Note that by $I^{ \pm *}(\mathbf{k})$ we mean $\left[I^{ \pm}(\mathbf{k})\right]^{*}$. Similar results are obtained for $I^{-}$and $J^{ \pm}$. Collectively these imply that $S^{v / b}$ and $S^{+}$are Hermitian (e.g., $\left.S_{i j}^{v}=S_{j i}^{v *}\right)$ while $S^{-}$is anti-Hermitian $\left(S_{i j}^{-}=-S_{j i}^{-*}\right)$. See Tables I and II.

The values of the correlations at zero separation correspond to bulk quantities (volume averages) of interest. These include the so-called ideal MHD rugged invariants $[13,44]$, which are used to characterize MHD turbulence phenomena (see, e.g., [45-47]). For 3D homogeneous MHD turbulence the rugged invariants are the total (kinetic plus magnetic) energy per unit mass $E^{t o t}=E^{k}+E^{m}=R_{i i}^{v}(0) / 2+R_{i i}^{b}(0) / 2$, the cross helicity $H_{c}=\langle\mathbf{v} \cdot \mathbf{b}\rangle / 2=R_{i i}^{+}(0) / 2$, and the magnetic helicity $H_{m}=\langle\mathbf{a} \cdot \nabla \times \mathbf{a}\rangle / 2$, where $\mathbf{b}=\nabla \times \mathbf{a}$. Other quadratic quantities of interest include the helicity of the velocity field $H_{k}=\langle\mathbf{v} \cdot \nabla \times \mathbf{v}\rangle$ (this is an invariant in 3D hydrodynamics, but is not thought to be rugged in the usual sense [48]) and the helicity of the electric current density $H_{j}=\langle\mathbf{j} \cdot \nabla \times \mathbf{j}\rangle$, which, although not an ideal invariant, rugged or otherwise, is of theoretical importance, in dynamo theory for example $[11,26]$. The helicities are usually connected with the indexantisymmetric portion of the correlation tensors (see Sec. $\mathrm{VI})$.

\section{APPENDIX B: SCALAR POTENTIALS}

Consider the solenoidal field $\mathbf{b}(\mathbf{x})=\nabla \times \mathbf{A}(\mathbf{x})$, so that in $k$ space $\mathbf{b}(\mathbf{k})=i \mathbf{k} \times \mathbf{A}(\mathbf{k})$. Working in a $k$-dependent coordinate system with an arbitrary uniform unit vector $\mathbf{e}$ and $a_{j}(\mathbf{k}), j=1,2,3$ the components of $\mathbf{A}$, we have

$$
\mathbf{A}(\mathbf{k})=a_{1} \mathbf{e}+i \frac{a_{2}}{k} \mathbf{k} \times \mathbf{e}+\frac{a_{3}}{k^{2}} \mathbf{k} \times(\mathbf{k} \times \mathbf{e}) .
$$

It is convenient to associate an $i$ with each $\mathbf{k}$ and in this form the $a$ 's all have the same dimensions. Thus

$$
\mathbf{b}(\mathbf{k})=i \mathbf{k} \times \mathbf{e}\left[a_{1}-a_{3}\right]-\mathbf{k} \times(\mathbf{k} \times \mathbf{e}) \frac{a_{2}}{k} .
$$

So there are really only two independent complex scalar potentials defining a solenoidal field since $a_{3}$ can be absorbed into $a_{1}$. This is clear geometrically since it only requires two independent vectors to span a plane perpendicular to $\mathbf{k}$. The two potentials generate the poloidal and toroidal components of the field (see, e.g., [11]). A customary mnemonic notation is $P(\mathbf{k})=a_{2}$ and $T(\mathbf{k})=a_{1}-a_{3}$. We refer to $P$ as the field's poloidal potential and $T$ as its toroidal potential. In $x$ space, where $P$ and $T$ are no longer dimensionally matched, we have

$$
\mathbf{b}(\mathbf{x})=-\nabla \times(\mathbf{e} \times \nabla P)-\mathbf{e} \times \nabla T .
$$


[1] H. P. Robertson, Proc. Camb. Philos. Soc. 36, 209 (1940).

[2] G. I. Taylor, Proc. R. Soc. London, Ser. A 151, 421 (1935).

[3] T. von Kármán and L. Howarth, Proc. R. Soc. London, Ser. A 164, 192 (1938).

[4] G. K. Batchelor, The Theory of Homogeneous Turbulence (Cambridge University Press, Cambridge, 1970).

[5] A. Monin and A. Yaglom, Statistical Fluid Mechanics (MIT Press, Cambridge, MA, 1971), Vol. 1; ibid. (MIT Press, Cambridge, MA, 1975), Vol. 2.

[6] G. K. Batchelor, Proc. R. Soc. London, Ser. A 186, 480 (1946).

[7] S. Chandrasekhar, Philos. Trans. R. Soc. London, Ser. A 242, 557 (1950).

[8] W. H. Matthaeus and C. Smith, Phys. Rev. A 24, 2135 (1981).

[9] S. Chandrasekhar, Proc. R. Soc. London, Ser. A 204, 435 (1951).

[10] S. Chandrasekhar, Proc. R. Soc. London, Ser. A 207, 301 (1951).

[11] F. Krause and K.-H. Rädler, Mean-Field Magnetohydrodynamics and Dynamo Theory (Akademie-Verlag, Berlin, 1980).

[12] H. K. Moffatt, Magnetic Field Generation in Electrically Conducting Fluids (Cambridge University Press, New York, 1978).

[13] U. Frisch, A. Pouquet, J. Léorat, and A. Mazure, J. Fluid Mech. 68, 769 (1975).

[14] W. H. Matthaeus and D. Montgomery, Ann. (N.Y.) Acad. Sci. 357, 203 (1980)

[15] W. H. Matthaeus, M. L. Goldstein, and C. W. Smith, Phys. Rev. Lett. 48, 1256 (1982).

[16] S. Chandrasekhar, Proc. R. Soc. London, Ser. A 203, 358 (1950).

[17] G. Rüdiger, Differential Rotation and Stellar Convection (Gordon and Breach, New York, 1989).

[18] W. H. Matthaeus, J. W. Bieber, and G. P. Zank, Rev. Geophys. Suppl. 33, 609 (1995).

[19] E. Marsch and C.-Y. Tu, J. Plasma Phys. 41, 479 (1989).

[20] Y. Zhou and W. H. Matthaeus, J. Geophys. Res. 95, 10291 (1990a).

[21] Multiple time correlations are not considered here, except to the extent that they may provide good estimates for spatial correlations when a uniform constant mean flow of sufficient strength rapidly sweeps fluctuations past a detector. However, this frozen-in flow property [2] permits only limited information about the full correlations to be obtained because it is generally not possible to obtain measurements corresponding to the full range of $\mathbf{r}$ (see Sec. VIII).

[22] S. A. Orszag, Stud. Appl. Math. 48, 275 (1969).

[23] R. Betchov, Phys. Fluids 4, 925 (1961).

[24] J. W. Belcher and L. Davis, Jr., J. Geophys. Res. 76, 3534 (1971).

[25] H. K. Moffatt and M. R. E. Proctor, Geophys. Astrophys. Fluid Dyn. 21, 265 (1982).

[26] H. K. Moffatt, J. Fluid Mech. 35, 117 (1969).

[27] M. A. Berger and G. B. Field, J. Fluid Mech. 147, 133 (1984).

[28] D. C. Montgomery and L. Turner, Phys. Fluids 24, 825 (1981).

[29] M. Lesieur, Turbulence in Fluids (Nijhoff, Dordrecht, The Netherlands, 1990).

[30] For example, $k_{i} \epsilon_{j \mu \nu} e_{\mu} k_{\nu}-k_{j} \epsilon_{i \mu \nu} e_{\mu} k_{\nu}=k^{2} \epsilon_{i j \alpha} e_{\alpha}-\mathbf{e} \cdot \mathbf{k} \epsilon_{i j \alpha} k_{\alpha}$ and $e_{i} \epsilon_{j \mu \nu} e_{\mu} k_{\nu}-e_{j} \epsilon_{i \mu \nu} e_{\mu} k_{\nu}=\mathbf{e} \cdot \mathbf{k} \epsilon_{i j \alpha} e_{\alpha}-\epsilon_{i j \alpha} k_{\alpha}$.
[31] See the remark in the paragraph below Eq. (19) for an explanation of the nonequivalence of Eq. (22) and the Fourier transform of Eq. (21).

[32] R. Aris, Vectors, Tensors, and the Basic Equations of Fluid Mechanics (Dover, Mineola, NY, 1989).

[33] Some care is necessary here since the notation is dense with " $H$ ' functions. Unlike the other $H$ 's, $H_{c}$ is not a scalar function associated with the antisymmetric part of a " $c$ " tensor but the cross helicity.

[34] D. C. Montgomery, Phys. Scr. T2/1, 83 (1982).

[35] G. P. Zank and W. H. Matthaeus, J. Plasma Phys. 48, 85 (1992a).

[36] J. V. Shebalin, W. H. Matthaeus, and D. Montgomery, J. Plasma Phys. 29, 525 (1983).

[37] M. Hossain, G. Vahala, and D. Montgomery, Phys. Fluids 28, 3074 (1985).

[38] V. Carbone and P. Veltri, Geophys. Astrophys. Fluid Dyn. 52, 153 (1990).

[39] S. Oughton, E. R. Priest, and W. H. Matthaeus, J. Fluid Mech. 280, 95 (1994b).

[40] J. W. Bieber, W. Wanner, and W. H. Matthaeus, J. Geophys. Res. 101, 2511 (1996).

[41] E. Marsch and C. Y. Tu, in Proceedings of Solar Wind 7, COSPAR Colloquium Series, edited by E. Marsch and R. Schwenn (Pergamon, Oxford, 1992), Vol. 3, p. 505.

[42] C.-Y. Tu, E. Marsch, and K. M. Thieme, J. Geophys. Res. 94, 11739 (1989a).

[43] The Dirac $\delta$ function on the left-hand side of Eq. (A5) arises because we are dealing with homogeneous turbulence in an infinite domain. Thus the integral of the absolute value of any nonzero function over the entire volume is unbounded. Consequently, it is necessary to use Stieltjes integrals instead of the more familiar Riemann ones (see, for example, Sec. 2.5 of Ref. [4]). This technicality may be sidestepped by working with velocity and magnetic fields whose integrals over all space are bounded, but which differ from the perfectly homogeneous fields only in physically unimportant ways. For example, take $\mathbf{v}$ and $\mathbf{b}$ to be periodic with very large wavelengths and use Fourier series; alternatively, suppose $\mathbf{v}$ and $\mathbf{b}$ are zero outside a very large box, permitting use of the standard Fourier integrals. In any case, for our purposes the distinction is unimportant and we hereafter drop the $\delta$ functions in such equations and use shorthand forms such as $S_{i j}^{v}(\mathbf{k})=\left\langle v_{i}(-\mathbf{k}) v_{j}(\mathbf{k})\right\rangle$ $=\left\langle v_{i}^{*}(\mathbf{k}) v_{j}(\mathbf{k})\right\rangle$.

[44] R. H. Kraichnan and D. C. Montgomery, Rep. Prog. Phys. 43, 547 (1980)

[45] W. H. Matthaeus and M. L. Goldstein, J. Geophys. Res. 87, 6011 (1982a).

[46] A. C. Ting, W. H. Matthaeus, and D. Montgomery, Phys. Fluids 29, 3261 (1986).

[47] T. Stribling and W. H. Matthaeus, Phys. Fluids B 3, 1848 (1991).

[48] R. H. Kraichnan, J. Fluid Mech. 59, 745 (1973).

[49] A. Pouquet, U. Frisch, and J. Léorat, J. Fluid Mech. 77, 321 (1976).

[50] R. Grappin, U. Frisch, J. Léorat, and A. Pouquet, Astron. Astrophys. 105, 6 (1982).

[51] R. Grappin, A. Pouquet, and J. Léorat, Astron. Astrophys. 126, 51 (1983). 